\title{
Shifted Symplectic Structures
}

\author{
T. Pantev*† \\ Department of Mathematics \\ University of Pennsylvania \\ M. Vaquié ${ }^{\dagger}$ \\ Institut de Math. de Toulouse \\ Université Paul Sabatiér
}

\author{
B. Toën ${ }^{\dagger}$ \\ I3M \\ Université de Montpellier2 \\ G. Vezzosi ${ }^{\dagger \dagger}$ \\ Institut Mathématique de Jussieu \\ Université Paris Diderot
}

March 2012

\begin{abstract}
This is the first of a series of papers about quantization in the context of derived algebraic geometry. In this first part, we introduce the notion of $n$-shifted symplectic structures ( $n$-symplectic structures for short), a generalization of the notion of symplectic structures on smooth varieties and schemes, meaningful in the setting of derived Artin $n$-stacks (see HAG-II, To2 ). We prove that classifying stacks of reductive groups, as well as the derived stack of perfect complexes, carry canonical 2-symplectic structures. Our main existence theorem states that for any derived Artin stack $F$ equipped with an $n$-symplectic structure, the derived mapping stack $\operatorname{Map}(X, F)$ is equipped with a canonical $(n-d)$-symplectic structure as soon a $X$ satisfies a CalabiYau condition in dimension $d$. These two results imply the existence of many examples of derived moduli stacks equipped with $n$-symplectic structures, such as the derived moduli of perfect complexes on Calabi-Yau varieties, or the derived moduli stack of perfect complexes of local systems on a compact and oriented topological manifold. We explain how the known symplectic structures on smooth moduli spaces of simple objects (e.g. simple sheaves on Calabi-Yau surfaces, or simple representations of $\pi_{1}$ of compact Riemann surfaces) can be recovered from our results, and that they extend canonically as 0 -symplectic structures outside of the smooth locus of simple objects. We also deduce new existence statements, such as the existence of a natural (-1)symplectic structure (whose formal counterpart has been previously constructed in Co,, $\left.\mathrm{Co}_{-\mathrm{Gw}}\right)$ on the derived mapping scheme $\operatorname{Map}\left(E, T^{*} X\right)$, for $E$ an elliptic curve and $T^{*} X$ is the total space of the cotangent bundle of a smooth scheme $X$. Canonical $(-1)$-symplectic structures are also shown to exist on Lagrangian intersections, on moduli of sheaves (or complexes of sheaves) on Calabi-Yau 3-folds, and on moduli of representations of $\pi_{1}$ of compact topological 3-manifolds. More generally, the moduli sheaves on higher dimensional varieties are shown to carry canonical shifted symplectic structures (with a shift depending on the dimension).
\end{abstract}

\footnotetext{
*Partially supported by NSF RTG grant DMS-0636606 and NSF grants DMS-0700446 and DMS1001693.

${ }^{\dagger}$ Partially supported by the ANR grant ANR-09-BLAN-0151 (HODAG).

${ }^{\ddagger}$ G. V. would like to thank the Insitute des Hautes Études Scientifiques (France) for providing perfect conditions for completing this paper.
} 


\section{Contents}

\begin{tabular}{l|l} 
Introduction & 2
\end{tabular}

1 Definitions and properties $\quad 12$

1.1 Graded mixed complexes . . . . . . . . . . . . . . . . 12

$1.2 p$-Forms, closed $p$-forms and $n$-shifted symplectic structures . . . . . . 17

2 Existence of shifted symplectic structures 29

2.1 Mapping stacks . . . . . . . . . . . . . . . . . . . . . 30

2.2 Lagrangian intersections . . . . . . . . . . . . . . . . . . 38

2.32 -shifted symplectic structure on $\mathbb{R}$ Perf . . . . . . . . . . . . . 41

3 Examples and applications 44

3.1 0-shifted symplectic structures on moduli of sheaves on curves and surfaces 44

3.2 (-1)-shifted symplectic structures and symmetric obstruction theories . . . 47

\section{Introduction}

This is the first part of a series of papers about quantization in the context of derived algebraic geometry, and specifically about the construction of quantized versions of various kinds of moduli spaces. In this article we start with the study of symplectic structures in the derived setting by introducing the notion of shifted symplectic structures of degree $n$ (or $n$-shifted symplectic structures) where $n \in \mathbb{Z}$ is an arbitrary integer. This is a direct and far reaching generalization of the notion of symplectic structures on smooth algebraic varieties and schemes (recovered when $n=0$ ), to the setting of derived and higher derived Artin stacks of [HAG-II, To2]. In this work we give a careful rigorous definition of $n$-shifted symplectic structures on derived Artin stacks (see Definition 1.18), and prove three existence theorems (see theorems 2.5, 2.9, 2.12) which provide powerful construction methods and many examples. This notion is an extension of the usual notion of symplectic structures on smooth schemes on the one hand to higher algebraic stacks and on the other hand to derived schemes and derived stacks. Based on these results, we recover some known constructions, such as the symplectic structures on various types of moduli spaces of sheaves on surfaces (see for instance [Mu, Hu-Le, In, In-Iw-Sa]) and the symmetric obstruction theories on moduli of sheaves on Calabi-Yau 3-folds (see [Be-Fa]), and prove new existence results, by constructing natural $n$-shifted symplectic structures on many other moduli spaces, including sheaves on higher dimensional varieties. These results may be summarized as follows.

Theorem 0.1 1. Let $X$ be a smooth and proper Calabi-Yau variety of dimension $d$. Then the derived moduli stack of perfect complexes of quasi-coherent sheaves on $X$ admits a canonical $(2-d)$-shifted symplectic structure.

2. Let $X$ be a smooth and proper variety of dimension $d$. Then, the derived moduli stack of perfect complexes with flat connections on $X$ admits a canonical $2(1-d)$-shifted symplectic structure. 
3. Let $M$ be a compact oriented topological manifold of dimension d. Then, the derived moduli stack of perfect complexes of local systems on $M$ admits a canonical $(2-d)$ shifted symplectic structure.

Future parts of this work will be concerned with the dual notion of Poisson (and $n$ Poisson) structures in derived algebraic geometry, formality (and $n$-formality) theorems, and finally with quantization.

\section{$p$-Forms, closed $p$-forms and symplectic forms in the derived setting}

A symplectic form on a smooth scheme $X$ (over some base ring $k$, of characteristic zero), is the datum of a closed 2-form $\omega \in H^{0}\left(X, \Omega_{X / k}^{2, c l}\right)$, which is moreover required to be non-degenerate, i.e. it induces an isomorphism $\Theta_{\omega}: T_{X / k} \simeq \Omega_{X / k}^{1}$ between the tangent and cotangent bundles. In our context $X$ will no longer be a scheme, but rather a derived Artin stack in the sense of HAG-II, To2, the typical example being an $X$ that is the solution to some derived moduli problem (e.g. of sheaves, or complexes of sheaves on smooth and proper schemes, see [To-Va, Corollary 3.31], or of maps between proper schemes as in [HAG-II, Corollary 2.2.6.14]). In this context, differential 1-forms are naturally sections in a quasi-coherent complex $\mathbb{L}_{X / k}$, called the cotangent complex (see [II, To2]), and the quasi-coherent complex of $p$-forms is defined to be $\wedge^{p} \mathbb{L}_{X / k}$. The $p$-forms on $X$ are then naturally defined as sections of $\wedge^{p} \mathbb{L}_{X / k}$, i.e. the set of $p$-forms on $X$ is defined to be the (hyper)cohomology group $H^{0}\left(X, \wedge^{p} \mathbb{L}_{X / k}\right)$. More generally, elements in $H^{n}\left(X, \wedge^{p} \mathbb{L}_{X / k}\right)$ are called $p$-forms of degree $n$ on $X$ (see Definition 1.12 and Proposition 1.14). The first main difficulty is to define the notion of closed $p$-forms and of closed $p$-forms of degree $n$ in a meaningful manner. The key idea of this work is to interpret $p$-forms, i.e. sections of $\wedge^{p} \mathbb{L}_{X / k}$, as functions on the derived loop stack $\mathcal{L} X$ of [To2, To-Ve-1, Ben-Nad] by means of the HKR theorem of [To-Ve-2] (see also [Ben-Nad]), and to interpret closedness as the condition of being $S^{1}$-equivariant. One important aspect here is that $S^{1}$-equivariance must be understood in the sense of homotopy theory, and therefore the closedness defined in this manner is not simply a property of a $p$-form but consists of an extra structure (see Definition 1.10). This picture is accurate (see Remark 1.9] and 1.16), but technically difficult to work with 1 . We have therefore chosen a different presentation, by introducing local constructions for affine derived schemes, that are then glued over $X$ to obtain global definitions for any derived Artin stack $X$. With each commutative dg-algebra $A$ over $k$, we associate a graded complex, called the weighted negative cyclic complex of $A$ over $k$, explicitly constructed using the derived de Rham complex of $A$. Elements of weight $p$ and of degree $n-p$ of this complex are by definition closed $p$-forms of degree $n$ on $\mathbf{S p e c} A$ (Definition 1.8). For a general derived Artin stack $X$, closed $p$-forms are defined by smooth descent techniques (Definition 1.12). This definition of closed $p$-forms has a more explicit local nature, but can be shown to coincide with the original idea of $S^{1}$-equivariant functions on the loop stack $\mathcal{L} X$ (using, for instance, results from [To-Ve-2, Ben-Nad]).

By definition a closed $p$-form $\omega$ of degree $n$ on $X$ has an underlying $p$-form of degree $n$ (as we already mentioned this underlying $p$-form does not determine the closed $p$-form

\footnotetext{
${ }^{1}$ One of the difficulties lies in the fact that we need to consider only functions formally supported around the constant loops $X \hookrightarrow \mathcal{L} X$, and this causes troubles because of the various completions involved.
} 
$\omega$, and several different closed $p$-form can have the same underlying $p$-form). When $p=2$ this underlying 2 -form is an element in $H^{n}\left(X, \wedge^{2} \mathbb{L}_{X / k}\right)$, and defines a natural morphism in the derived category of quasi-coherent complexes on $X$

$$
\Theta_{\omega}: \mathbb{T}_{X / k} \longrightarrow \mathbb{L}_{X / k}[n]
$$

where $\mathbb{T}_{X / k}$ is the tangent complex (i.e. the dual of $\mathbb{L}_{X / k}$ ). With this notation in place, we give the main definition of this paper:

Definition 0.2 An $n$-shifted symplectic form on a derived Artin stack $X$ is a closed 2 -form $\omega$ of degree $n$ on $X$ such that the corresponding morphism

$$
\Theta_{\omega}: \mathbb{T}_{X / k} \longrightarrow \mathbb{L}_{X / k}[n]
$$

is an isomorphism in the quasi-coherent derived category $D_{q c o h}(X)$ of $X$.

When $n=0$ and $X$ is a smooth $k$-scheme, the definition above recovers the usual notion of a symplectic structure, and nothing more. Smooth schemes do not admit $n$-shifted symplectic structures for $n \neq 0$, but there are many interesting examples of 0 -shifted symplectic structures on derived Artin stacks (see corollaries 2.6, 2.13, see also [Pe]). Therefore, not only the above definition provides an extension of the notion of symplectic structure by introducing the parameter $n$, but even for $n=0$ the notion of 0 -shifted symplectic structure is a new way to extend the notion of symplectic structures on non-smooth schemes.

An $n$-shifted symplectic form $\omega$ can be thought of as the data consisting of a quasiisomorphism

$$
\Theta: \mathbb{T}_{X / k} \longrightarrow \mathbb{L}_{X / k}[n]
$$

together with an entire hierarchy of higher coherences expressing some subtle relations between $\Theta$ and the differential geometry of $X$. The quasi-isomorphism $\Theta$ can itself be understood as a kind of duality between the stacky part of $X$, expressed in the nonnegative part of $\mathbb{T}_{X}$, and the derived part of $X$, expressed in the non-positive part of $\mathbb{L}_{X / k}$ (this is striking already when $n=0$, and this picture has to be qualified when $n$ is far away from 0 ). In practice, when $X$ is some moduli of sheaves on some space $M$, this duality is often induced by a version of Poincaré duality (or Serre duality) on $M$, since tangent complexes are then expressed in terms of the cohomology of $M$. It is tempting to view $n$-shifted symplectic structures as a non-abelian incarnation of Poincaré duality, which is definitely a good way to think about them in the context of non-abelian cohomology (see the paragraph Related works at the end of this Introduction).

\section{Existence results}

In this paper we prove three existence results for $n$-shifted symplectic structures. To start with, we show that classifying stacks $B G$, for reductive affine group schemes $G$, are naturally endowed with 2 -shifted symplectic structures. The underlying 2 -form here is clear, it is given by the degree 2 shift of a non-degenerate $G$-invariant quadratic form:

$$
\mathfrak{g}[1] \wedge \mathfrak{g}[1] \simeq \operatorname{Sym}^{2}(\mathfrak{g})[2] \longrightarrow k[2],
$$


where $\mathfrak{g}$ is the Lie algebra of $G$ over $k$. The fact that this 2 -form can be naturally promoted to a closed 2-form on $B G$ follows from the simple observation that all 2-forms on $B G$ are canonically closed - geometrically this is due to the fact that the space of functions on $\mathcal{L} B G$ is discrete, and thus $S^{1}$ acts on it in a canonically trivial manner.

Our first existence result is the following

Theorem 0.3 The derived stack $\mathbb{R}$ Perf of perfect complexes of quasi-coherent sheaves is equipped with a natural 2-shifted symplectic form.

The relation between the above theorem and the 2-shifted symplectic form on $B G$ is given by the canonical open embedding $B G L_{n} \subset \mathbb{R}$ Perf, sending a vector bundle to the corresponding perfect complex concentrated in degree 0: the 2-shifted symplectic form on $\mathbb{R}$ Perf restricts to the one on $B \mathrm{GL}_{n}$. The proof of Theorem 0.3 uses the Chern character for perfect complexes, with values in negative cyclic homology. The weight 2 part of the Chern character of the universal perfect complex on $\mathbb{R}$ Perf provides a canonical 2-form of degree 2 , which is non-degenerate by inspection.

The second existence theorem we prove in this paper is a transfer of $n$-shifted symplectic structures on a given derived Artin stack $F$ to the derived mapping stack $\operatorname{Map}(X, F)$, under certain orientability condition on $X$. This statement can be viewed as an algebraic version of the AKSZ-formalism (see [AKSZ] for the original reference), further extended to the setting of derived Artin stacks.

Theorem 0.4 Let $X$ be a derived stack endowed with an $\mathcal{O}$-orientation of dimension $d$, and let $(F, \omega)$ be a derived Artin stack with an n-shifted symplectic structure $\omega$. Then the derived mapping stack $\boldsymbol{M a p}(X, F)$ carries a natural $(n-d)$-shifted symplectic structure.

The condition of having an $\mathcal{O}$-orientation of dimension $d$ (see Definition 2.4) essentially means that $D_{q c o h}(X)$ satisfies the Calabi-Yau condition in dimension $d$. The typical example is of course when $X$ is a smooth and proper Calabi-Yau scheme (or DeligneMumford stack) of dimension $d$ (relative to Spec $k$ ). Other interesting examples are given, for instance, by de Rham or Dolbeault homotopy types $\left(Y_{D R}, Y_{D o l}\right.$ in the notation of [Si1] of a smooth and proper scheme $Y$ over $k$, for which $\operatorname{Map}\left(Y_{D R}, F\right)$, or $\operatorname{Map}\left(Y_{D o l}, F\right)$, should be understood as maps with flat connections, or with Higgs fields, from $Y$ to $F$. Theorem 0.3 and 0.4 provide many examples of $n$-shifted symplectic forms on moduli spaces of perfect complexes on Calabi-Yau schemes, or flat perfect complexes, or perfect complexes with Higgs fields, etc. The proof of Theorem 0.4 is rather natural, though the details require some care. We use the evaluation morphism

$$
X \times \operatorname{Map}(X, F) \longrightarrow F,
$$

to pull-back the $n$-shifted symplectic form on $F$ to a closed 2-form of degree $n$ on $X \times \operatorname{Map}(X, F)$. This closed 2-form is then integrated along $X$, using the $\mathcal{O}$-orientation (this is a quasi-coherent integration, for which we need Serre duality), in order to get a closed 2-form of degree $(n-d)$ on $\operatorname{Map}(X, F)$. Then, we observe that this last 2-form is non-degenerate. A large part of the argument consists of defining properly the integration map (see Definition 2.3).

Finally, our third and last existence statement concerns symplectic intersections and symplectic forms induced on them. For this we introduce the notion of a Lagrangian 
structure on a morphism $L \longrightarrow X$, where $X$ is equipped with an $n$-shifted symplectic form; this is a generalization of the notion of Lagrangian submanifolds (a closed immersion $L \hookrightarrow X$ of smooth schemes possesses a Lagrangian structure if and only if $L$ is Lagrangian in $X$ in the usual sense, and moreover this structure, if it exists, is unique).

Theorem 0.5 Let $(X, \omega)$ be a derived Artin stack with an n-shifted symplectic form $\omega$, and let

$$
L \longrightarrow X, \quad L^{\prime} \longrightarrow X
$$

be two morphisms of derived Artin stacks endowed with Lagrangian structures. Then, the derived fiber product $L \times_{X}^{h} L^{\prime}$ carries a natural $(n-1)$-shifted symplectic form.

As a corollary, we see that the derived intersection of two Lagrangian smooth subschemes $L, L^{\prime} \subset X$, into a symplectic smooth scheme $X$, always carries a natural $(-1)$ shifted symplectic structure.

Before going further we would like to mention here that the use of derived stacks in Theorems 0.3, 0.4, 0.5 is crucial, and that the corresponding results do not hold in the underived setting. The reason for this is that if a derived Artin stack $F$ is endowed with an $n$-shifted symplectic form $\omega$, then the pull-back of $\omega$ to the truncation $h^{0}(F)$ is a closed 2 -form of degree $n$ which is, in general, highly degenerate.

\section{Examples and applications}

The three theorems 0.3 , 0.4 and 0.5 listed above, imply the existence of many interesting and geometrically relevant examples of $n$-shifted symplectic structures. For instance, let $Y$ be a smooth and proper Deligne-Mumford stack with connected geometric fibers of relative dimension $d$.

1. The choice of a fundamental class $[Y] \in H_{D R}^{2 d}(Y, \mathcal{O}) 2$ in de Rham cohomology (relative to $\operatorname{Spec} k)$ determines a canonical $2(1-d)$-shifted symplectic form on the derived stack

$$
\mathbb{R} \operatorname{Perf}_{D R}(Y):=\operatorname{Map}\left(Y_{D R}, \mathbb{R} \text { Perf }\right)
$$

of perfect complexes with flat connections on $Y$.

2. The choice of a fundamental class $[Y] \in H_{D o l}^{2 d}(Y, \mathcal{O}) \sqrt{3}$ in Dolbeault cohomology (relative to $\operatorname{Spec} k)$ determines a canonical $2(1-d)$-shifted symplectic form on the derived stack

$$
\mathbb{R} \operatorname{Perf}_{D o l}(Y):=\operatorname{Map}\left(Y_{D o l}, \mathbb{R} \text { Perf }\right)
$$

of perfect complexes with Higgs fields.

\footnotetext{
${ }^{2}$ This stands for de Rham cohomology of $Y$ with coefficients in the trivial flat bundle $\mathcal{O}_{Y}$. It can be computed as usual, by taking hypercohomology of $Y$ with coefficients in the de Rham complex.

${ }^{3}$ This stands for Dolbeault cohomology of $Y$ with coefficients in the trivial Higgs bundle $\mathcal{O}_{Y}$. It can be computed as usual, by taking hypercohomology of $Y$ with coefficients in the Dolbeault complex.
} 
3. The choice of a trivialization (when it exists) $\omega_{Y / k} \simeq \mathcal{O}_{Y}$, determines a canonical $(2-d)$-shifted symplectic form on the derived stack

$$
\mathbb{R} \operatorname{Perf}(Y):=\operatorname{Map}(Y, \mathbb{R} \operatorname{Perf})
$$

of perfect complexes on $Y$.

4. If $M$ is a compact, orientable topological manifold of dimension $d$, then a choice of a fundamental class $[M] \in H_{d}(M, k)$ determines a canonical $(2-d)$-shifted symplectic form on the derived stack

$$
\mathbb{R P e r f}(M):=\operatorname{Map}(M, \mathbb{R P e r f})
$$

of perfect complexes on $M 4$.

We note here that the derived stack of perfect complexes of quasi-coherent sheaves on $Y$ considered above contains interesting open substacks, such as the stack of vector bundles, or the stack of simple objects. We use this observation, and our existence theorems, to recover in a new and uniform way, some well known symplectic structures on smooth moduli spaces of simple vector bundles (see [Mu, In]), and on character varieties (see [Go, Je]). One corollary of our results states that these known symplectic structures in fact extend to 0-shifted symplectic structures on the ambient derived Artin stacks, and this explains what is happening to the symplectic structures at the boundaries of these smooth open substacks, i.e. at bad points (vector bundles or representations with many automorphisms, or with non-trivial obstruction map, etc.).

As another application of our existence results we present a construction of symmetric obstruction theories, in the sense of [Be-Fa], by showing that a (-1)-shifted symplectic structure on a derived Artin stack $X$ always endows the truncation $h^{0}(X)$ with a natural symmetric obstruction theory. This enables us to construct symmetric obstruction theories on the moduli stack of local systems on a compact topological 3-manifold, or on the moduli stack of simple perfect complexes on a Calabi-Yau 3-fold. The latter result was recently used by Brav-Bussi-Dupont-Joyce ([Br-Bu-Du-Jo $]$ ) to prove that the coarse moduli space of simple perfect complexes of coherent sheaves, with fixed determinant, on a CalabiYau 3-fold admits, locally for the analytic topology, a potential, i.e. it is isomorphic to the critical locus of a function. This was a longstanding problem in Donaldson-Thomas theory. We remark that, as shown recently by Pandharipande-Thomas ( $\mathrm{Pa}-\mathrm{Th}])$, such a result is false for a general symmetric obstruction theory. Hence the existence of a local potential depends in a crucial way the existence of a global (-1)-shifted symplectic structure on the derived moduli stack of simple perfect complexes on a Calabi-Yau 3-fold. The results in Br-Bu-Du-Jo] suggest that one should have general formal, local-analytic and perhaps étale local versions of the Darboux theorem for (-1)-shifted - and maybe even for general $n$-shifted - symplectic forms. It will be interesting to compare such formal (-1)-shifted Darboux theorem with the formal potential defined in [Ko-So, Section 3.3]. Local structure theorems of this type also hint at the existence of Donaldson-Thomas theory for CalabiYau manifolds of higher dimensions. This is a completely unexplored territory with the

\footnotetext{
${ }^{4}$ A perfect complex on $M$ is by definition a complex of sheaves of $k$-modules locally quasi-isomorphic to a constant and bounded complex of sheaves made of projective $k$-modules of finite type.
} 
first case being the case of 4 -folds, where the corresponding derived moduli space carries a (-2)-symplectic structure by Theorem 0.3 .

Another interesting (-1)-shifted symplectic form whose existence follows from our Theorem 0.4 is the one obtained on $\operatorname{Map}\left(E, T^{*} X\right)$, where $E$ is an elliptic curve over Spec $k, X$ is a smooth $k$-scheme, and $T^{*} X$ is the total space of the cotangent bundle of $X$ (relative to $k$ ) equipped with its canonical symplectic structure. At the formal completion level this (-1)-shifted symplectic form on $\operatorname{Map}\left(E, T^{*} X\right)$ was constructed and studied in $[\mathrm{Co}$. A nice feature of our construction is that it produces this symplectic structure directly as a global form on the derived scheme $\operatorname{Map}\left(E, T^{*} X\right)$. Specifically we have

Corollary 0.6 Let $E$ be an oriented elliptic curve over $k$ with a fixed algebraic volume form, and let $X$ be a smooth k-scheme. Then the derived mapping scheme $\operatorname{Map}\left(E, T^{*} X\right)$ carries a canonical (-1)-shifted symplectic structure.

\section{Future works and open questions}

In a sequel to this paper, we will study the dual notion of $n$-Poisson structures on derived Artin stacks. This dual notion is technically more delicate to handle than the $n$-shifted symplectic structures discussed in this paper. This is essentially due to the fact that it is a much less local notion. Nevertheless we can follow the same reasoning and extract the notion of an $n$-Poisson structure from the geometry of derived loop stacks and higher loop stacks $\mathbb{L}^{(n)} X:=\operatorname{Map}\left(S^{n}, X\right)$. As usual $n$-shifted symplectic structures give rise to $n$-Poisson structures, and correspond precisely to the non-degenerate $n$-Poisson structures. Without going into technical details one can say that an $n$-Poisson structure on a derived Artin stack $X$ consists of the data of a bivector of degree $-n$

$$
\mathcal{P} \in H^{-n}\left(X, \wedge^{2}\left(\mathbb{T}_{X / k}[n]\right)[-2 n]\right) \simeq H^{-n}\left(X, \phi_{n}^{2}\left(\mathbb{T}_{X / k}\right)\right)
$$

where

$$
\phi_{n}^{2}\left(\mathbb{T}_{X / k}\right) \simeq\left\{\begin{array}{cc}
\wedge^{2}\left(\mathbb{T}_{X / k}\right) & \text { if } n \text { is even } \\
\operatorname{Sym}^{2}\left(\mathbb{T}_{X / k}\right) & \text { if } n \text { is odd }
\end{array},\right.
$$

together with higher coherences expressing a closedness conditions. Now, it turns out that the complex

$$
\mathbb{R} \Gamma\left(X, \operatorname{Sym}\left(\mathbb{T}_{X / k}[-n-1]\right)\right)[n+2]
$$

can be identified with the tangent complex of the deformation problem for the dg-category $L_{\text {qcoh }}(X)$ of quasi-coherent sheaves considered as an $n$-fold monoidal dg-category 5 : $n=0$ simply means as a dg-category, $n=1$ as a monoidal dg-category, $n=2$ as a braided monoidal dg-category, and so on. A higher version of Kontsevich formality theorem implies that an $n$-Poisson structure $\mathcal{P}$ defines an element in this tangent complex, satisfying a homotopy version of the master equation, and thus a formal deformation of $L_{q c o h}(X)$ considered as an $n$-fold monoidal dg-category. This formal deformation is, by definition, the quantization of $X$ with respect to the $n$-Poisson structure $\mathcal{P}$. As a consequence, if

\footnotetext{
${ }^{5}$ We can make sense of this also for negative $n$, by shifting the formal deformation variable in degree $-2 n$. For the moment, we will assume $n \geq 0$ in order to simplify the presentation.
} 
$X$ is endowed with an $n$-shifted symplectic structure, then it has a canonical quantization, which is, by definition, a formal deformation of $L_{q c o h}(X)$ as an $n$-fold monoidal dg-category. This will be our approach to construct quantizations of the derived moduli stacks on which, in this paper, we have constructed $n$-shifted symplectic structures. At the moment, this program is very much in progress, and two main difficulties remain. First of all the deformation theory of dg-categories and $n$-fold monoidal dg-categories has not been fully worked out in the literature. Even the case $n=0$ is not fully understood, as explained in [Ke-Lo and [Lu2, Remark 5.3.38]. We hope to make progress on this deformation theory by introducing the new machinery of tame homological algebra, and tame dg-categories. Ongoing work (see the forthcoming [To-Va-Ve] in that direction seems to provide a complete answer for $n=0$. We hope that similar ideas will also work for arbitrary $n$. The second issue concerns the higher version of Kontsevich formality theorem mentioned above, which states that the natural Lie bracket on the complex $\mathbb{R} \Gamma\left(X, \operatorname{Sym}\left(\mathbb{T}_{X / k}[-n-1]\right)\right)[n+1]$ coming from the fact that it is the tangent complex of the deformation problem of $n$-fold monoidal dg-categories, is the natural one (i.e. equals an appropriate version of the Schouten bracket). This higher version of the formality theorem is, at present, still a conjecture, even for the case $n=0$ and $X$ a general derived Artin stack (the only established case is $n=0$ and $X$ a smooth scheme).

\section{Related works}

There are many related works that should be mentioned here but for space reasons we will only discuss a small selection of such works.

To start with, our notion of 0-shifted symplectic structure generalizes the usual notion of symplectic structures on smooth schemes to the setting of derived Artin stacks. In this context the 0 -shifted symplectic structures provide a new point of view on symplectic structures over non-smooth objects, and a comparison with the notion of symplectic singularities and symplectic resolutions [Kal, Kal-Le-So, Na, Ne-McG] would certainly be interesting. We note however that 0 -shifted symplectic structures cannot exist on singular schemes. This is due to the fact that for the cotangent complex to be its own dual we need some non-trivial stacky structure. Therefore, 0 -shifted symplectic structures do not bring anything new for singularities of schemes, but are surely interesting for singularities appearing on some moduli stacks and on their coarse moduli spaces. As noted in [Fu, Thm. 6.1], there exist coarse moduli spaces of sheaves on K3 surfaces having symplectic singularities but with no symplectic resolution. However, these moduli spaces are coarse moduli of natural derived Artin stacks, and according to our results these derived stacks carry natural 0 -shifted symplectic structures. It is tempting to consider the derived Artin stack itself as a symplectic resolution of its coarse moduli space.

We have explained how several well known symplectic structures can be recovered from our existence theorem. Similar symplectic structures are known to exist on moduli of certain sheaves on non Calabi-Yau manifolds (see for instance $\mathrm{Ku}-\mathrm{Ma}$ ). We believe that these can also be recovered from a slight modification of our constructions, but we will not pursue this direction in the present work.

Symplectic structures also appear in non-commutative geometry, in particular on moduli spaces of sheaves on non-commutative Calabi-Yau varieties. More generally, our existence Theorem 2.12 has an extension to the case of the derived stack $\mathcal{M}_{T}$ of objects in a 
Calabi-Yau dg-category $T$, constructed in To-Va]: the derived stack $\mathcal{M}_{T}$ carries a natural $(2-d)$-shifted symplectic structure when $T$ is a Calabi-Yau dg-category of dimension $d$. The proof of this non-commutative extension of Theorem 2.12 is very close to the proof of our Theorem 2.5. but is not included in this work. In another direction, we think that sheaves on non-commutative Calabi-Yau varieties of fractional dimension should also carry a suitable version of shifted symplectic structures.

Many of the $n$-shifted symplectic structures we construct in this paper live on derived moduli stacks of bundles (or complexes of bundles) with flat, or Higgs structures $\left(\mathbb{R} \operatorname{Loc}_{D R}(X), \mathbb{R} L o c_{D o l}(X)\right.$, etc.). These are the moduli stacks appearing in non-abelian Hodge theory, and in fact, the derived moduli of flat perfect complexes $\mathbb{R} \operatorname{Perf}_{D R}(X)$ is used in [Si2] to construct the universal non-abelian Hodge filtration. The $n$-shifted symplectic forms on these moduli stacks reflects the Poincaré duality in de Rham (or Dolbeault, or Betti) cohomology, and this should be thought of as an incarnation of Poincaré duality in non-abelian cohomology. They give important additional structures on these moduli stacks, and are expected to play an important role for the definition of polarizations in non-abelian Hodge theory.

Our Theorem 0.4 should be viewed as an algebraic version of one of the main construction in AKSZ. Similarly, some of the constructions and notions presented here are very close to constructions and notions introduced in $\mathrm{Co}$. More precisely, our construction and results about degree $(-1)$ derived symplectic structures might be seen as a globalization of Costello's formal derived (in the sense of [Lu2]) approach. A complete comparison would require $\mathcal{C}^{\infty}$ and complex analytic versions of derived algebraic geometry, and a notion of $n$-shifted symplectic structures in such contexts. We are convinced that most, if not all, the definitions and results we present in this work have $\mathcal{C}^{\infty}$ and complex analytic analogs. Derived differential and complex analytic geometries do exist thanks to [Lu3, but going through the notions of forms, closed forms and symplectic structures in these settings is not completely straightforward.

Finally, the reader will notice that most of the methods we give in this work provide $m$ shifted symplectic structures starting from already existing $n$-shifted symplectic structures with $n>m$ (e.g. Theorem 2.5] and 2.9) . It would also be interesting to have constructions that increase the degree of symplectic structures. The 2-shifted symplectic forms on $B G$ and $\mathbb{R}$ Perf are of this kind, but it would be interesting to have general methods for constructing $n$-shifted symplectic forms on quotients, dual to the one we have on fiber products and on mapping stacks.

Acknowledgements. We are thankful to K.Costello for sharing his ideas on ( -1$)$-shifted symplectic forms with us, as well as to D.Calaque and G.Ginot for conversations on $E_{n^{-}}$ deformation theory and the higher dimensional formality conjecture. We are grateful to D.Joyce for sending us a draft of [Br-Bu-Du-Jo], where (-1)-shifted symplectic forms were used to establish the existence of a local analytic potential in Donaldson-Thomas theory for Calabi-Yau 3-folds; this was a great happy ending to our paper. We also thank F.Bonechi, A.Cattaneo, D.Kaledin, D.Kazhdan, M.Kontsevich, D.Maulik, F.Paugam and B.Tsygan for stimulating conversations on the subject while we were writing this paper. We finally thank P-E.Paradan for pointing out the reference [Je]. 


\section{Notations and conventions.}

- $k$ is a base commutative ring, noetherian and of residual characteristic zero.

- $d g_{k}$ is the category of dg-modules over $k$ (i.e. of complexes of $k$-modules). By convention, the differential of an object in $d g_{k}$ increases degrees. For an object $E \in d g_{k}$, we will sometimes use the notation

$$
\pi_{i}(E):=H^{-i}(E)
$$

- $c d g a_{k}$ is the category of commutative dg-algebras over $k$, and $c d g a_{k}^{\leq 0}$ its full subcategory of non-positively graded commutative dg-algebras.

- $d g_{k}, c d g a_{k}$ (respectively $c d g a_{k}^{\leq 0}$ ) are endowed with their natural model structures for which equivalences are quasi-isomorphisms, and fibrations are epimorphisms (respectively epimorphisms in strictly negative degrees).

- $d A f f_{k}:=\left(c d g a_{k}^{\leq 0}\right)^{o p}$ is the category of derived affine $k$-schemes.

- The expression $\infty$-category will always refer to $(\infty, 1)$-category (see $[\mathrm{Ber}]$ ). To fix ideas we will use Segal categories as models for $\infty$-categories (see [Si3], and [To-Ve-1, $\S 1])$.

- The $\infty$-categories associated to the model categories $d g_{k}, c d g a_{k}^{\leq 0}, d A f f_{k}$ are denoted by $\operatorname{dg}_{k}, \operatorname{cdga}_{k}^{\leq 0}, \mathbf{d A f f}_{k}$.

- The $\infty$-category of simplicial sets is denoted by $\mathbb{S}$. It is also called the $\infty$-category of spaces, and space will be used to mean simplicial set.

- The $\infty$-category of derived stacks over $k$, for the étale topology, is denoted by $\mathbf{d S t}_{k}$ (see [To2, HAG-II]). If $X$ is a derived stack, the $\infty$-category of derived stacks over $X$ is denoted by $\mathbf{d S t}_{X}$. The truncation of a derived stack $X$ is denoted by $h^{0}(X)$. The derived mapping stack between $X$ and $Y$ is denoted by $\mathbb{R} \operatorname{Map}(X, Y)$.

- We will use the expressions homotopy limits and $\infty$-limits interchangeably to refer either to homotopy limits in an ambient model category, or to limits in an ambient $\infty$-category. The same convention will be used for colimits. Homotopy fiber products will be denoted as usual by $X \times{ }_{Z}^{h} Y$.

- The mapping space between two objects $a$ and $b$ in an $\infty$-category $A$ is denoted by $\operatorname{Map}_{A}(a, b)$. Points in $\operatorname{Map}_{A}(a, b)$ will be called morphisms in $A$, and paths in $\operatorname{Map}_{A}(a, b)$ homotopies. A morphism in an $\infty$-category will be called an equivalence if it is a homotopy equivalence (i.e. becomes an isomorphism in the homotopy category). The word equivalence will also refer to a weak equivalence in a model category.

- A derived Artin stack is by definition a derived stack which is $m$-geometric for some integer $m$, for the étale topology and the class $\mathbf{P}$ of smooth maps (see [HAG-II, §2.2.2] and [To2]). All derived Artin stacks are assumed to be locally of finite presentation over Spec $k$. 
- For a derived stack $X$, its quasi-coherent derived category is denoted by $D_{q c o h}(X)$, and its $\infty$-categorical version by $L_{q c o h}(X)$. The larger derived category of all $\mathcal{O}_{X^{-}}$ modules is denoted by $D\left(\mathcal{O}_{X}\right)$, and its $\infty$-categorical version by $L\left(\mathcal{O}_{X}\right)$. By definition, $L_{q c o h}(X)$ is a full sub- $\infty$-category of $L\left(\mathcal{O}_{X}\right)$. As usual, morphisms between $x$ and $y$ in $D_{q c o h}(X)$ will be denoted by $[x, y]$. We have

$$
[x, y] \simeq \pi_{0}\left(\operatorname{Map}_{L_{q c o h}(X)}(x, y)\right) .
$$

We refer to [To2, To1], and also [Lu3], for detailed definitions and properties of these $\infty$-categories.

- Complexes of morphisms between objects $x$ and $y$ in $L\left(\mathcal{O}_{X}\right)$ or $L_{q c o h}(X)$ will be denoted by $\mathbb{R} \underline{\operatorname{Hom}}(x, y)$. The $\infty$-categories $L\left(\mathcal{O}_{X}\right)$ and $L_{q c o h}(X)$ have natural symmetric monoidal structures (in the sense of [To-Ve-1, Lu5]), and this monoidal structures are closed. The internal mapping object in $L\left(\mathcal{O}_{X}\right)$ between $x$ and $y$ is denoted by $\mathbb{R} \underline{\mathcal{H} o m}(x, y)$. In particular, for an object $x$, we denote by $x^{\vee}:=\mathbb{R} \underline{\mathcal{H} o m}\left(x, \mathcal{O}_{X}\right)$ its dual. Perfect complexes on $X$ are by definition dualizable objects in $L_{q c o h}(X)$.

- Expressions such as $f^{*}, f_{*}$ and $\otimes_{k}$ should be understood in the derived sense, and should be read as $\mathbb{L} f^{*}, \mathbb{R} f_{*}, \otimes_{k}^{\mathbb{L}}$, unless specified otherwise.

- For a derived Artin stack $X$, we denote by $\mathbb{L}_{X / k} \in L_{q c o h}(X)$ its cotangent complex over Spec $k$ (see [To2, HAG-II] and [Lu5, §7.3]). Since, according to our conventions, $X$ is assumed to be locally of finite presentation, it follows that $\mathbb{L}_{X / k}$ is a perfect complex on $X$, and thus is dualizable. Its dual is denoted by $\mathbb{T}_{X / k}:=\mathbb{L}_{X / k}^{\vee}$ and is called the tangent complex of $X$ over Spec $k$.

\section{Definitions and properties}

In this first section we give the definitions of $p$-forms, closed $p$-forms and symplectic forms over a derived Artin stack. We will start by some elementary constructions in the setting of mixed and graded mixed complexes. These constructions will then be applied to define $p$-forms and closed $p$-forms over an affine derived scheme Spec $A$, by using some explicit graded mixed complexes constructed from the derived de Rham complex of $A$. Finally, these constructions are shown to be local for the smooth topology and to glue on smooth covers, giving global notions for any derived Artin stack. This smooth descent property is not a completely obvious statement, and its proof requires some care.

\subsection{Graded mixed complexes}

Recall from [Ka] that a mixed complex over $k$ is a dg-module over $k[\epsilon]=H_{*}\left(S^{1}, k\right)$, where $\epsilon$ is in degree -1 and satisfies $\epsilon^{2}=0$. The category of mixed complexes will be denoted by $\epsilon-d g_{k}$, and also called the category of $\epsilon$-dg-modules over $k$. The differential of the complex of $k$-modules underlying an object $E \in \epsilon-d g_{k}$ will be denoted by $d$, and by convention it raises degrees $d: E^{n} \longrightarrow E^{n+1}$.

The tensor product $\otimes_{k}$ makes $\epsilon-d g_{k}$ into a symmetric monoidal category, which is moreover a symmetric monoidal model category for which the weak equivalences are the 
quasi-isomorphisms (see [To-Ve-2, §2]). By definition a graded mixed complex over $k$ is a mixed complex $E$ over $k$, equipped with a direct sum decomposition of the underlying complex of $k$-dg-modules

$$
E:=\bigoplus_{p \in \mathbb{Z}} E(p)
$$

in such a way that multiplication by $\epsilon$ has degree 1

$$
\epsilon: E(p) \longrightarrow E(p+1)
$$

while the differential $d$ of the complex of $k$-modules underlying $E$ respects this grading

$$
d: E(p) \longrightarrow E(p) .
$$

The extra grading will be called the weight grading, and we will say that elements in $E(p)$ have weight $p$.

Graded mixed complexes over $k$ form a category denoted by $\epsilon-d g_{k}^{g r}$, that is again a symmetric monoidal model category, for the tensor product over $k$ with the usual induced grading

$$
\left(E \otimes_{k} F\right)(p):=\bigoplus_{i+j=p} E(i) \otimes_{k} E(j),
$$

and with weak equivalences being the quasi-isomorphisms.

Remark 1.1 Alternatively, graded mixed complexes can be viewed as dg-comodules over the commutative dg-Hopf-algebra (see also [Ben-Nad]

$$
B_{\epsilon}:=H^{*}\left(\mathbb{G}_{m} \ltimes B \mathbb{G}_{a}, \mathcal{O}\right),
$$

the cohomology Hopf algebra of the semi-direct product group stack $\mathbb{G}_{m} \ltimes B \mathbb{G}_{a}$. It is the semi-direct (or cross-)product of the multiplicative Hopf algebra $k\left[t, t^{-1}\right]$ with $k[e]=$ $H^{*}\left(S^{1}, k\right)$, via the natural action of $\mathbb{G}_{m}$ on $k[e]$ given by rescaling $e$. More precisely, as a commutative dg-algebra, $B_{\epsilon}$ is $k\left[t, t^{-1}\right] \otimes_{k} k[e]$, with zero differential and comultiplication determined by

$$
\Delta(t)=t \otimes t \quad \Delta(e)=t \otimes e .
$$

Note that the tautological equivalence of symmetric monoidal categories

$$
\epsilon-d g_{k}^{g r} \simeq B_{\epsilon}-d g-\operatorname{comod},
$$

commutes with the two forgetful functors to the category of complexes of $k$-dg-modules.

Let $E \in \epsilon-d g_{k}$ be a mixed complex over $k$. We may form the usual negative cyclic object $N C(E)$, which is a dg-module over $k$ defined in degree $n$ by the formula

$$
N C^{n}(E):=\prod_{i \geq 0} E^{n-2 i}
$$

For an element $\left\{m_{i}\right\}_{i} \in \prod_{i \geq 0} E^{n-2 i}$, the differential is defined by the formula

$$
D\left(\left\{m_{i}\right\}\right)_{j}=\epsilon m_{j-1}+d m_{j} .
$$


If $E$ is moreover a graded mixed complex, then the extra grading $E=\bigoplus_{p} E(p)$ defines a sequence of sub-complexes $N C(E)(p) \subset N C(E)$, by

$$
N C^{n}(E)(p):=\prod_{i \geq 0} E^{n-2 i}(p+i) .
$$

These sub-complexes are natural direct summands (i.e the inclusions admit natural retractions), and thus we have natural morphisms

$$
\bigoplus_{p} N C(E)(p) \longrightarrow N C(E) \longrightarrow \prod_{p} N C(E)(p)
$$

These are monomorphisms of complexes, but in general they are neither isomorphisms nor quasi-isomorphisms.

The above constructions are functorial in $E$, and define a functor

$$
N C: \epsilon-d g_{k}^{g r} \longrightarrow d g_{k},
$$

from the category of graded mixed complexes to the category of complexes. Moreover, the functors $E \mapsto N C(E)(p)$ defines direct summands of the functor $N C$, together with natural transformations $\bigoplus_{p} N C(p) \longrightarrow N C \longrightarrow \prod_{p} N C(p)$. We will be interested in the family of functors $N C(p): \epsilon-d g_{k}^{g r} \longrightarrow d g_{k}$, as well as in their direct sum

$$
N C^{w}:=\bigoplus_{p} N C(p): \epsilon-d g_{k}^{g r} \longrightarrow d g_{k}^{g r}
$$

where $d g_{k}^{g r}$ denotes the category of graded complexes of $k$-modules - i.e. complexes of $k$-modules equipped with an extra $\mathbb{Z}$-grading, with morphisms the maps of complexes preserving the extra grading.

Definition 1.2 For a graded mixed complex $E$, the weighted negative cyclic complex is defined by

$$
N C^{w}(E):=\bigoplus_{p \in \mathbb{Z}} N C(E)(p) \in d g_{k}^{g r}
$$

Its cohomology is called the weighted negative cyclic homology of $E$, and is denoted by

$$
N C_{n}^{w}(E):=H^{-n}\left(N C^{w}(E)\right) \quad N C_{n}^{w}(E)(p):=H^{-n}\left(N C^{w}(E)(p)\right) .
$$

The natural decomposition

$$
N C_{n}^{w}(E) \simeq \bigoplus_{p \in \mathbb{Z}} N C_{n}^{w}(E)(p)
$$

is called the Hodge decomposition.

As observed above, we have a natural morphism

$$
N C^{w}(E) \longrightarrow N C(E)
$$

which is, in general, not a quasi-isomorphism. The complex $N C(E)$ computes the usual negative cyclic homology of the mixed complex $E$, which can differ from the weighted cyclic 
homology as defined above. The two coincide under obvious boundedness conditions on E.

Both categories $\epsilon-d g_{k}^{g r}$ and $d g_{k}^{g r}$ have natural projective model structures, where weak equivalences are quasi-isomorphisms and fibrations are epimorphisms, on the underlying complexes of $k$-modules (see [To-Ve-2]).

Proposition 1.3 The functor

$$
N C^{w}: \epsilon-d g_{k}^{g r} \longrightarrow d g_{k}^{g r}
$$

is a right Quillen functor for the projective model structures.

Proof. We denote by $k(p) \in \epsilon-d g_{k}^{g r}$ the graded mixed complex consisting of $k$ sitting in degree 0 and having pure weight $p$. We construct an explicit cofibrant replacement $Q(p)$ of $k(p)$ as follows. As a graded $k[\epsilon]$-module, $Q(p)$ is

$$
Q(p)=\bigoplus_{j \geq 0} k[\epsilon][2 j]=k[\epsilon]\left[a_{0}, \ldots, a_{j}, \ldots\right]
$$

where $a_{j}$ stands for the canonical generator of $k[\epsilon][2 j]$ (in degree $-2 j$ ). The differential in $Q(p)$ is then given by

$$
d a_{j}+\epsilon \cdot a_{j-1}=0
$$

Finally, $a_{j}$ is declared to be of weight $p+j$. The natural projection

$$
Q(p) \longrightarrow k(p)
$$

sending all the $a_{j}$ 's to zero for $j>0$, and $a_{0}$ to $1 \in k$, is a quasi-isomorphism of graded mixed complexes. Moreover $Q(p)$ is a free graded module over $k[\epsilon]$, and thus is a cofibrant object in $\epsilon-d g_{k}^{g r}$. Finally, we have

$$
N C^{w}(E)(p) \simeq \underline{\operatorname{Hom}}(Q(p), E),
$$

where $\underline{H o m}$ stands for the complex of morphisms between two objects in $\epsilon-d g_{k}^{g r}\left(\epsilon-d g_{k}^{g r}\right.$ is a $C(k)$-model category in a natural way, where $C(k)$ is the symmetric monoidal model category of complexes of $k$-modules). This finishes the proof of the proposition.

Since all objects in the model categories $\epsilon-d g_{k}^{g r}$ and $d g_{k}^{g r}$ are fibrant for the projective model structures, Proposition 1.3 implies in particular that $N C^{w}$ preserves quasiisomorphisms. Following our conventions, we will denote by

$$
\mathbf{d g}_{k}^{g r}, \quad \epsilon-\mathbf{d g}_{k}, \quad \text { and } \quad \epsilon-\mathbf{d g}_{k}^{g r},
$$

the $\infty$-categories obtained by Dwyer-Kan localization from the corresponding model categories $d g_{k}^{g r}, \epsilon-d g_{k}$ and $\epsilon-d g_{k}^{g r}$. The functor $N C^{w}$ thus defines an $\infty$-functor

$$
N C^{w}=\bigoplus_{p} N C^{w}(p): \epsilon-\mathbf{d g}_{k}^{g r} \longrightarrow \mathbf{d g}_{k}^{g r}
$$


Corollary 1.4 The $\infty$-functor

$$
N C^{w}: \epsilon-\mathbf{d g}_{k}^{g r} \longrightarrow \mathbf{d g}_{k}^{g r}
$$

preserves $\infty$-limits. Moreover, we have natural equivalences

$$
N C^{w}(E)(p) \simeq \mathbb{R} \underline{\operatorname{Hom}}(k(p), E)
$$

where $k(p) \in \epsilon-d g_{k}^{g r}$ is the graded mixed complex consisting of $k$ sitting in degree 0 and having pure weight $p$.

Proof. This is a consequence of the statement and proof of proposition 1.3.

Remark 1.5 The notion of weighted negative cyclic homology has the following geometric interpretation (see also Ben-Nad]). We let the multiplicative group scheme $\mathbb{G}_{m}$ (over Spec $k$ ) act on the group stack $B \mathbb{G}_{a}$, and form the semi-direct product group stack $\mathbb{G}_{m} \ltimes$ $B \mathbb{G}_{a}$. It can be shown from the definitions that there exists an equivalence of $\infty$-categories

$$
L_{q c o h}\left(B\left(\mathbb{G}_{m} \ltimes B \mathbb{G}_{a}\right)\right) \simeq \epsilon-\mathbf{d g}_{k}^{g r} .
$$

This equivalence is obtained by sending a quasi-coherent complex $E$ on the stack $B\left(\mathbb{G}_{m} \ltimes\right.$ $\left.B \mathbb{G}_{a}\right)$ to its fiber at the base point Spec $k \longrightarrow B\left(\mathbb{G}_{m} \ltimes B \mathbb{G}_{a}\right)$, which is naturally equipped with an action of $\mathbb{G}_{m} \ltimes B \mathbb{G}_{a}$, and thus a structure of a comodule over the dg-coalgebra of cochains $C^{*}\left(\mathbb{G}_{m} \ltimes B \mathbb{G}_{a}, \mathcal{O}\right)$. This dg-coalgebra turns out to be formal and quasiisomorphic to the dg-coalgebra $B_{\epsilon}$ discussed above - the semi-direct product of $k\left[t, t^{-1}\right]$ with $k[e]$. This fiber is therefore a graded mixed complex, and it is straightforward to check that this construction induces an equivalence of $\infty$-categories as stated. Using this point of view, the $\infty$-functor $N C^{w}$ has the following interpretation. Consider the natural projection

$$
\pi: B\left(\mathbb{G}_{m} \ltimes B \mathbb{G}_{a}\right) \longrightarrow B \mathbb{G}_{m} .
$$

It induces a direct image on the $\infty$-categories of quasi-coherent complexes

$$
\pi_{*}: L_{q c o h}\left(B\left(\mathbb{G}_{m} \ltimes B \mathbb{G}_{a}\right)\right) \longrightarrow L_{q c o h}\left(B \mathbb{G}_{m}\right),
$$

right adjoint to the pull-back functor

$$
\pi^{*}: L_{q c o h}\left(B \mathbb{G}_{m}\right) \longrightarrow L_{q c o h}\left(B\left(\mathbb{G}_{m} \ltimes B \mathbb{G}_{a}\right)\right) .
$$

If we identify $L_{q c o h}\left(B\left(\mathbb{G}_{m} \ltimes B \mathbb{G}_{a}\right)\right)$ with $\epsilon-\mathbf{d g}_{k}^{g r}$ as above, and $L_{q c o h}\left(B \mathbb{G}_{m}\right)$ with $\mathbf{d g}_{k}^{g r}$, then $\pi_{*}$ becomes isomorphic to $N C^{w}$. In other words, $N C^{w}(E)$ computes the homotopy fixed point of $E$ under the action of $B \mathbb{G}_{a}$. The residual $\mathbb{G}_{m}$-action then corresponds to the grading by the pieces $N C^{w}(E)(p)$.

We finish this section by observing that the graded complex $N C^{w}(E)$ comes equipped with a natural projection

$$
N C^{w}(E) \longrightarrow E
$$


which is functorial in $E$ and is a morphism of graded complexes. It consists of the morphisms induced by the projection to the $i=0$ component

$$
N C^{w}(E)^{n}(p)=\prod_{i \geq 0} E^{n-2 i}(p+i) \longrightarrow E^{n}(p)
$$

Remark 1.6 If $E$ is a graded $\epsilon$-dg-module (i.e a graded mixed complex) over $k$, then for any weight $p$ there is a natural map - that might be called the absolute $\epsilon$-differential -

$$
D_{E}(p): E(p) \longrightarrow \mathrm{NC}^{w}(E)(p+1)[-1]
$$

whose degree $m$ piece is

$$
D_{E}^{m}(p): E^{m}(p) \rightarrow \prod_{i \geq 0} E^{m-2 i-1}(p+1+i): x_{m, p} \mapsto\left(\epsilon x_{m, p}, 0,0, \ldots\right) .
$$

\section{$1.2 p$-Forms, closed $p$-forms and $n$-shifted symplectic structures}

Let $A \in c d g a_{k}^{\leq 0}$ be a commutative differential non-positively graded algebra over $k$, and let us denote by $\Omega_{A / k}^{1}$ the $A$-dg-module of Kähler differential 1-forms of $A$ over $k$. We can form its de Rham algebra over $k$ (see [To-Ve-2])

$$
D R(A / k):=\operatorname{Sym}_{A}\left(\Omega_{A / k}^{1}[1]\right),
$$

where in contrast with our usual usage $S y m_{A}$ here refers to the underived symmetric product of $A$-dg-modules. The complex $D R(A / k)$ is in a natural way a commutative $\epsilon$ dg-algebra in the sense of [To-Ve-2, §2]. Here we will consider $D R(A / k)$ just as a graded mixed complex, by forgetting the extra multiplicative structure. The underlying complex of $k$-modules is simply

$$
\operatorname{Sym}_{A}\left(\Omega_{A / k}^{1}[1]\right) \simeq \bigoplus_{p} \Omega_{A / k}^{p}[p]
$$

where $\Omega_{A / k}^{p}:=\wedge_{A}^{p} \Omega_{A / k}^{1}$. The mixed structure is induced by the de Rham differential $\epsilon:=d_{D R}: \Omega_{A / k}^{p} \longrightarrow \Omega_{A / k}^{p+1}$, and is the unique mixed structure on $\operatorname{Sym}_{A}\left(\Omega_{A / k}^{1}[1]\right)$ making it into an $\epsilon$-cdga and for which the action of $\epsilon$ on the factor $A=\wedge_{A}^{0} \Omega_{A / k}^{1}$ is the universal dg-derivation $d: A \longrightarrow \Omega_{A / k}^{1}$ (see [To-Ve-2, $\left.\S 2\right]$ ). Finally, the grading on $D R(A / k)$ is the one for which $D R(A / k)(p):=\Omega_{A / k}^{p}[p]$. This graded mixed structure on $D R(A / k)$ is also compatible with the multiplicative structure, and makes it into a graded mixed cdga over $k$, but we will not make use of this finer structure in this work.

The assignment $A \mapsto D R(A / k)$ defines a functor

$$
c d g a_{k}^{\leq 0} \longrightarrow \epsilon-d g_{k}^{g r} .
$$

This functor can be derived on the left, by pre-composing it with a cofibrant replacement functor on $c g d a_{k}^{\leq 0}$, to obtain

$$
\mathbb{L} D R(-/ k): c d g a_{k}^{\leq 0} \longrightarrow \epsilon-d g_{k}^{g r}
$$


which now preserves quasi-isomorphisms. Therefore, it induces a well defined $\infty$-functor $\mathbf{D R}(-/ k): \mathbf{c d g a}_{k} \longrightarrow \epsilon-\mathbf{d g}_{k}^{g r}$. Recall that, for $A$ a cdga over $k$, the underlying complex of $\mathbf{D R}(A / k)$ is

$$
\bigoplus_{p}\left(\wedge_{A}^{p} \mathbb{L}_{A / k}\right)[p]
$$

where $\mathbb{L}_{A / k}$ is the cotangent complex of $A$ over $k$, and $\wedge_{A}^{p}$ must now be understood in the derived sense (see the proof of [To-Ve-2, Proposition 2.4]).

Definition 1.7 Let $A \in \mathbf{c d g a}_{k}$. The weighted negative cyclic complex of $A$ over $k$ is defined by

$$
N C^{w}(A / k):=N C^{w}(\mathbf{D R}(A / k)) .
$$

This defines an $\infty$-functor

$$
N C^{w}: \operatorname{cdga}_{k} \longrightarrow \mathbf{d g}_{k}^{g r} .
$$

As we have seen, for any graded mixed complex $E$, we have a natural projection

$$
N C^{w}(E) \longrightarrow E
$$

which is a morphism of graded complexes. We get this way, for any $p \geq 0$, a natural morphism of complexes

$$
N C^{w}(A / k)(p) \longrightarrow \wedge_{A}^{p} \mathbb{L}_{A / k}[p] .
$$

For a complex of $k$-modules $E$, we will denote by $|E|$ the simplicial set obtained by the Dold-Kan correspondence (applied to the truncation $\tau_{\leq 0}(E)$ ). By definition we have a natural weak equivalence $|E| \simeq \operatorname{Map}_{\mathbf{d g}_{k}}(k, E)$, where $M a p_{\mathbf{d g}_{k}}$ denotes the mapping space (i.e. simplicial set) in the $\infty$-category $\mathbf{d g}_{k}$. For $A \in \mathbf{c d g a}_{k}$, and two integers $p \geq 0$ and $n \in \mathbb{Z}$, we set

$$
\mathcal{A}_{k}^{p}(A, n):=\left|\wedge_{A}^{p} \mathbb{L}_{A / k}[n]\right| \in \mathbb{S} .
$$

This defines an $\infty$-functor $\mathcal{A}_{k}^{p}(-, n): \mathbf{c d g a}_{k} \longrightarrow \mathbb{S}$. In the same way, we set

$$
\mathcal{A}_{k}^{p, c l}(A, n):=\left|N C^{w}(A / k)[n-p](p)\right| .
$$

Using the natural projection mentioned above $N C^{w}(A / k)[n-p](p) \longrightarrow \wedge^{p} \mathbb{L}_{A / k}[n]$, we deduce a natural morphism $\mathcal{A}_{k}^{p, c l}(A, n) \longrightarrow \mathcal{A}_{k}^{p}(A, n)$. We have therefore two $\infty$-functors

$$
\mathcal{A}_{k}^{p, c l}(-, n), \mathcal{A}_{k}^{p}(-, n): \operatorname{cdga}_{k} \longrightarrow \mathbb{S},
$$

together with a natural transformation $\mathcal{A}_{k}^{p, c l}(-, n) \longrightarrow \mathcal{A}_{k}^{p}(-, n)$.

Definition 1.8 For $A \in \mathbf{c d g a}_{k}$, the simplicial set $\mathcal{A}_{k}^{p}(A, n)$ (respectively $\mathcal{A}_{k}^{p, c l}(A, n)$ ) is called the space of $p$-forms of degree $n$ on the derived stack $\operatorname{Spec} A$, relative to $k$ (respectively the space of closed $p$-forms of degree $n$ on the derived stack $\mathbf{S p e c} A$, relative to $k)$.

As usual, when the ground ring $k$ is clear from the context, we will simply write $\mathcal{A}^{p}(-, n)$ and $\mathcal{A}^{p, c l}(-, n)$ for $\mathcal{A}_{k}^{p}(-, n)$ and $\mathcal{A}_{k}^{p, c l}(-, n)$. 
Remark 1.9 We have seen that graded mixed complexes can be understood as quasicoherent complexes on the stack $B \mathcal{H}$, where $\mathcal{H}$ is the semi-direct product group stack $\mathbb{G}_{m} \ltimes B \mathbb{G}_{a}$ (see our remark 1.5). If we continue this point of view, $p$-forms and closed $p$-forms can be interpreted as follows (see also [Ben-Nad]).

Let $X=\operatorname{Spec} A$ be a derived affine scheme over $k$, and consider its derived loop stack $\mathcal{L} X:=\mathbb{R} \operatorname{Map}\left(S^{1}, X\right)$. The natural morphism $S^{1}=B \mathbb{Z} \longrightarrow B \mathbb{G}_{a}$, induced by the inclusion $\mathbb{Z} \hookrightarrow \mathbb{G}_{a}$, induces a morphism

$$
\mathcal{L}^{u} X:=\mathbb{R} \operatorname{Map}\left(B \mathbb{G}_{a}, X\right) \longrightarrow \mathcal{L} X
$$

This morphism turns out to be an equivalence of derived schemes. Therefore, the group stack $\mathcal{H}$ of automorphisms of $B \mathbb{G}_{a}$, acts naturally on $\mathcal{L} X$. We can form the quotient stack and consider the natural projection

$$
p:[\mathcal{L} X / \mathcal{H}] \longrightarrow B \mathcal{H}
$$

Using the results of [To-Ve-2] it is possible to show that there exists a functorial equivalence in $L_{q c o h}(B \mathcal{H})$

$$
p_{*}\left(\mathcal{O}_{[\mathcal{L} X / \mathcal{H}]}\right) \simeq \mathbf{D R}(A / k),
$$

where $\mathbf{D R}(A / k)$ is viewed as an object in $L_{q c o h}(B \mathcal{H})$ using our remark 1.5,

As $p$-forms and closed $p$-forms are defined directly from the graded mixed complexes, this explains the precise relation between our notion of closed $p$-forms, and functions on derived loop stacks. For instance, we have that $N C^{w}(A / k)$ are simply the $B \mathbb{G}_{a}$-invariants (or equivalently the $S^{1}$-invariants through $S^{1} \longrightarrow B \mathbb{G}_{a}$ ) in the complex of functions on $\mathcal{L} X$, in the sense that we have a natural equivalence of quasi-coherent sheaves on $B \mathbb{G}_{m}$

$$
q_{*}\left(\mathcal{O}_{[\mathcal{L} X / \mathcal{H}]}\right) \simeq N C^{w}(A / k),
$$

where now $q$ is the projection $[\mathcal{L} X / \mathcal{H}] \longrightarrow B \mathcal{H} \longrightarrow B \mathbb{G}_{m}$.

Note that the space $\mathcal{A}^{p, c l}(A, n)$, of closed $p$-forms of some degree $n$, is not a full subspace (i.e. not a union of connected components) of $\mathcal{A}^{p}(A, n)$. For a point $w \in \mathcal{A}^{p}(A, n)$, the homotopy fiber $K(w)$ of the map $\mathcal{A}^{p, c l}(A, n) \longrightarrow \mathcal{A}^{p}(A, n)$, taken at $w$, can be a complicated space. Contrary to what the terminology closed p-forms seems to suggest, being closed is not a well defined property for a $p$-form, and there is indeed an entire space of "closing structures" on a given $p$-form, namely the homotopy fiber $K(w)$. As a point in $K(w)$ consists of the data needed to "close" the $p$-form $w$, we will call $K(w)$ the space of keys of $w$. For future reference we record this in the following definition.

Definition 1.10 For $A \in \mathbf{c d g a}_{k}$, and $w \in \mathcal{A}^{p}(A, n)$, the space $K(w)$, is defined to be the homotopy fiber of the natural map $\mathcal{A}^{p, c l}(A, n) \longrightarrow \mathcal{A}^{p}(A, n)$ taken at $w$. It is called the space of keys of $w$.

We have defined two $\infty$-functors $\mathcal{A}^{p}(-, n), \quad \mathcal{A}^{p, c l}(-, n): \mathbf{c d g a}_{k} \longrightarrow \mathbb{S}$ that we consider as derived pre-stacks, $\mathbf{d A f f}_{k}^{o p} \longrightarrow \mathbb{S}$. When $A \in \mathbf{c d g a}_{k}$ is viewed as a derived scheme $X=\operatorname{Spec} A$, we will obviously write

$$
\mathcal{A}^{p}(X, n)=\mathcal{A}^{p}(A, n) \quad \mathcal{A}^{p, c l}(X, n)=\mathcal{A}^{p, c l}(A, n) .
$$


Proposition 1.11 The derived pre-stacks $\mathcal{A}^{p}(-, n)$ and $\mathcal{A}^{p, c l}(-, n)$ are derived stacks for the étale topology.

Proof. For $\mathcal{A}^{p}(-, n)$, we have by definition

$$
\mathcal{A}^{p}(\operatorname{Spec} A, n) \simeq \operatorname{Map}_{\mathbf{d g}_{k}}\left(k,\left(\wedge_{A}^{p} \mathbb{L}_{A / k}\right)[n]\right) .
$$

Therefore, the fact that $\mathcal{A}^{p}(-, n)$ satisfies étale descent follows from the fact that the functor Spec $A \mapsto \wedge^{p} \mathbb{L}_{A / k}$ satisfies étale descent. This latter $\infty$-functor, when restricted to the small étale site of a derived affine scheme $X$ is a quasi-coherent complex of $\mathcal{O}_{X^{-}}$ modules, and thus is a derived stack for the étale (and in fact the fpqc) topology (see [HAG-II, Lemma 2.2.2.13]).

In the same way, the derived pre-stack

$$
\operatorname{Spec} A \mapsto \mathbf{D R}(A / k)[n-p] \simeq \bigoplus_{q}\left(\wedge_{A}^{q} \mathbb{L}_{A / k}\right)[q-p+n]
$$

is a quasi-coherent complex on the small étale site of $X=\operatorname{Spec} A$, and so satisfies étale descent. Therefore, Corollary 1.4 implies that

$$
\operatorname{Spec} A \mapsto N C^{w}(\mathbf{D R}(A / k)[n-p]) \simeq N C^{w}(\mathbf{D R}(A / k))[n-p]
$$

is a derived stack for the étale topology. Taking the degree $p$ part and applying the Dold-Kan correspondence, we deduce that $\operatorname{Spec} A \mapsto \mathcal{A}^{p, c l}(A, n)$ has descent for the étale topology.

Proposition 1.11 enables us to globalize the definition of $\mathcal{A}^{p}(X, n)$ and $\mathcal{A}^{p, c l}(X, n)$ to any derived stack $X$, as follows

Definition 1.12 Let $F \in \mathbf{d S t}_{k}$ be a derived stack over $k, p$ and $n$ integers with $p \geq 0$. The space of $p$-forms, relative to $k$, of degree $n$ on $F$ is defined by

$$
\mathcal{A}_{k}^{p}(F, n):=\operatorname{Map}_{\mathbf{d S t}_{k}}\left(F, \mathcal{A}_{k}^{p}(-, n)\right) .
$$

The space of closed $p$-forms, relative to $k$, of degree $n$ on $F$ is defined by

$$
\mathcal{A}_{k}^{p, c l}(F, n):=\operatorname{Map}_{\mathbf{d S t}_{k}}\left(F, \mathcal{A}_{k}^{p, c l}(-, n)\right) .
$$

As before, when the base ring $k$ is clear from the context, we will not include it in the notation.

Using the natural projection $\mathcal{A}^{p, c l}(-, n) \longrightarrow \mathcal{A}^{p}(-, n)$, we have, for any $F$, a natural projection $\mathcal{A}^{p, c l}(F, n) \longrightarrow \mathcal{A}^{p}(F, n)$.

Definition 1.12 above has an alternative description, based on Corollary 1.4. Consider the derived pre-stack Spec $A \mapsto \mathbf{D R}(A / k)$. It is a derived pre-stack with values in $\epsilon-\mathbf{d g}_{k}^{g r}$. As the forgetful $\infty$-functor

$$
\epsilon-\mathbf{d g}_{k}^{g r} \longrightarrow \mathbf{d g}_{k}^{g r}
$$

is conservative and preserves $\infty$-limits, Corollary 1.4 implies that $\mathbf{D R}$ is a derived stack with values in graded mixed complexes. By left Kan extension, this derived stack extends uniquely to an $\infty$-functor (see e.g. [To-Ve-1, §1.2] or [Lu1])

$$
\mathbf{D R}(-/ k): \mathbf{d S t}_{k}^{o p} \longrightarrow \epsilon-\mathbf{d g}_{k}^{g r} .
$$

Therefore, as in Definition 1.12, we may give the following 
Definition 1.13 For a derived stack $F$ we set

$$
N C^{w}(F / k):=N C^{w}(\mathbf{D R}(F / k)) \in \mathbf{d g}_{k}^{g r} .
$$

As usual, if $k$ is clear from the context, we will write

$$
\mathbf{D R}(F)=\mathbf{D R}(F / k) \quad N C^{w}(F)=N C^{w}(F / k) .
$$

Note that Corollary 1.4 implies that we have natural equivalences

$$
\mathcal{A}_{k}^{p}(F, n) \simeq|\mathbf{D R}(F / k)[n-p](p)| \quad \mathcal{A}_{k}^{p, c l}(F, n) \simeq\left|N C^{w}(F / k)[n-p](p)\right| .
$$

The second of these equivalences is sometimes useful to compute the spaces of closed $p$ forms by first computing explicitly the graded mixed complex $\mathbf{D R}(F / k)$ and then applying the $\infty$-functor $N C^{w}$.

The space of closed $p$-forms on a general derived stack $F$ can be a rather complicated object, even when $F$ is a nice derived Artin stack. The space of $p$-forms, however, has the following, expected, description.

Proposition 1.14 Let $F$ be a derived Artin stack over $k$, and $\mathbb{L}_{F / k} \in L_{q c o h}(F)$ be its cotangent complex relative to $k$. Then, we have an equivalence

$$
\mathcal{A}^{p}(F, n) \simeq \operatorname{Map}_{L_{q c o h}(F)}\left(\mathcal{O}_{F}, \wedge^{p} \mathbb{L}_{F / k}[n]\right) .
$$

This equivalence is functorial in $F$ in the obvious sense. In particular, we have a functorial bijective map

$$
\pi_{0}\left(\mathcal{A}^{p}(F, n)\right) \simeq H^{n}\left(F, \wedge^{p} \mathbb{L}_{F / k}\right) .
$$

Proof. We start by constructing a morphism

$$
\phi_{F}: \operatorname{Map}_{L_{q c o h}(F)}\left(\mathcal{O}_{F}, \wedge^{p} \mathbb{L}_{F / k}[n]\right) \longrightarrow \mathcal{A}^{p}(F, n),
$$

functorial in $F$. By definition, the right hand side is given by

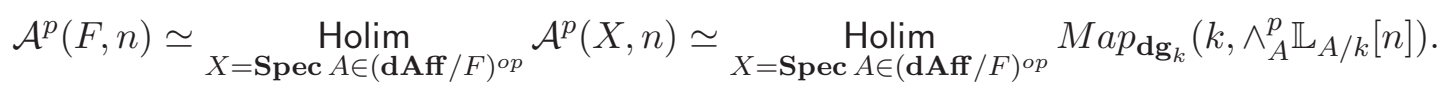

On the $\infty$-site dAff $/ F$, of derived affine schemes over $F$, we have a (non-quasi-coherent) $\mathcal{O}$-module, denoted by $\wedge^{p} \mathbb{L}$, and defined by

$$
X=\operatorname{Spec} A \mapsto \wedge_{A}^{p} \mathbb{L}_{A / k}
$$

We also have the quasi-coherent $\mathcal{O}$-module $\wedge^{p} \mathbb{L}_{F / k}$. There exists a natural morphism of $\mathcal{O}$-modules on dAff $/ F, \wedge^{p} \mathbb{L}_{F / k} \longrightarrow \wedge^{p} \mathbb{L}$. obtained, over $u:$ Spec $A \longrightarrow F$, by the natural morphism $u^{*}\left(\mathbb{L}_{F / k}\right) \longrightarrow \mathbb{L}_{A / k}$. This defines a morphism on global sections

$$
\operatorname{Map}_{L_{q c o h}(F)}\left(\mathcal{O}_{F}, \wedge^{p} \mathbb{L}_{F / k}[n]\right) \longrightarrow \underset{X=\operatorname{Spec} A \in(\mathbf{d A f f} / F)^{o p}}{\operatorname{Holim}_{A a p}} \operatorname{Map}_{\mathbf{d g}_{k}}\left(k, \wedge_{A}^{p} \mathbb{L}_{A / k}[n]\right) \simeq \mathcal{A}^{p}(F, n) .
$$

We will now check that this morphism

$$
\phi: \operatorname{Map}_{L_{q c o h}(F)}\left(\mathcal{O}_{F}, \wedge^{p} \mathbb{L}_{F / k}[n]\right) \longrightarrow \mathcal{A}^{p}(F, n)
$$


is an equivalence. For this we assume that $F$ is a derived Artin stack which is $m$-geometric for some $m \geq 0$ (see [HAG-II, $\$ 1.3 .3]$ ), and we proceed by induction on $m$. For $m=0$, $F$ is a derived affine stack and the statement is true. We assume that the statement is correct for $(m-1)$-geometric derived stacks. We can write $F$ as the quotient of a smooth Segal groupoid object $X_{*}$, with $X_{i}$ being $(m-1)$-geometric for all $i$ (see [HAG-II, §1.3.4]).

We have a commutative square of descent maps

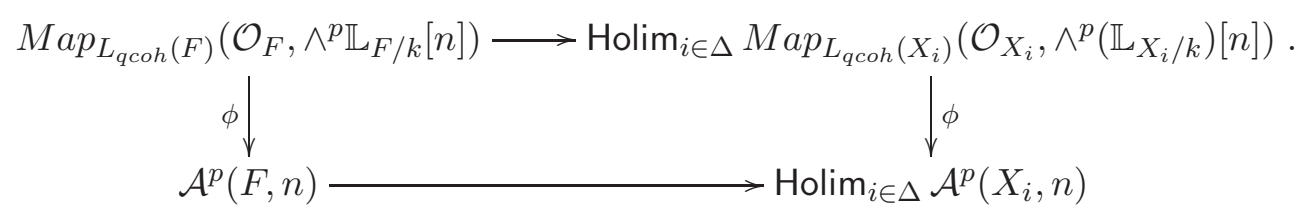

By induction, the right vertical morphism between the homotopy limits is an equivalence. The bottom horizontal morphism is also an equivalence, because $F \mapsto \mathcal{A}^{p}(F, n)$ sends $\infty$-colimits to $\infty$-limits by definition. It thus remains to show that the top horizontal morphism is an equivalence. But this follows from the following lemma.

Lemma 1.15 Let $X_{*}$ be a smooth Segal groupoid object in derived Artin stacks, with quotient $F=\left|X_{*}\right|$. Then, for any integer $p$ and $n$, the natural morphism

$$
\operatorname{Map}_{L_{q c o h}(F)}\left(\mathcal{O}_{F}, \wedge^{p} \mathbb{L}_{F / k}[n]\right) \longrightarrow \operatorname{Holim}_{i \in \Delta} \operatorname{Map}_{L_{q c o h}\left(X_{i}\right)}\left(\mathcal{O}_{X_{i}}, \wedge^{p} \mathbb{L}_{X_{i} / k}[n]\right)
$$

is an equivalence.

Proof of lemma - For any $n$, the morphisms appearing in the statement of the lemma are retracts of the natural morphism

$\operatorname{Map}_{L_{q c o h}(F)}\left(\mathcal{O}_{F}, \bigoplus_{p}\left(\wedge^{p} \mathbb{L}_{F / k}\right)[n-p]\right) \rightarrow \operatorname{Holim}_{i \in \Delta}\left(\operatorname{Map}_{L_{q c o h}\left(X_{i}\right)}\left(\mathcal{O}_{X_{i}}, \bigoplus_{p}\left(\wedge^{p} \mathbb{L}_{X_{i} / k}\right)[n-p]\right)\right)$

The mapping spaces of the $\infty$-category $L_{\text {qcoh }}(F)$ are related to the derived Hom's by the formula

$$
\operatorname{Map}_{L_{q c o h}(F)}(E, F) \simeq|\mathbb{R} \underline{\operatorname{Hom}}(E, F)|,
$$

and therefore it is enough to prove that

$$
\mathbb{R} \underline{\operatorname{Hom}}\left(\mathcal{O}_{F}, \bigoplus_{p}\left(\wedge^{p} \mathbb{L}_{F / k}\right)[-p]\right) \longrightarrow \operatorname{Holim}_{i \in \Delta} \underline{\mathbb{R o m}}\left(\mathcal{O}_{X_{i}}, \bigoplus_{p}\left(\wedge^{p} \mathbb{L}_{X_{i} / k}\right)[-p]\right)
$$

is a quasi-isomorphism of complexes of $k$-modules.

For a derived stack $F$, we denote by $T^{1}(F)$ the shifted tangent stack

$$
T^{1}(F):=\operatorname{Map}\left(\operatorname{Spec} k\left[e_{-1}\right], F\right),
$$

where $e_{-1}$ is in degree -1 . The $\infty$-functor $F \mapsto T^{1}(F)$ preserves derived Artin stacks and finite homotopy limits. Moreover, if $X \longrightarrow Y$ is a smooth and surjective morphism of derived Artin stacks then $T^{1}(X) \longrightarrow T^{1}(Y)$ is an epimorphism of derived stacks, as one may see by using the infinitesimal criterion for smoothness [HAG-II, $§ 2.2 .5$ ]. It follows 
formally from these properties that the natural morphism $\left|T^{1} X_{*}\right| \longrightarrow T^{1}\left|X_{*}\right|=T^{1}(F)$ is an equivalence. This implies that we have

$$
\underline{\mathbb{H o m}}_{L_{q c o h}\left(T^{1}(F)\right)}\left(\mathcal{O}_{T^{1}(F)}, \mathcal{O}_{T^{1}(F)}\right) \simeq \operatorname{Holim}_{i \in \Delta} \mathbb{R} \underline{\operatorname{Hom}}_{L_{q c o h}\left(T^{1}\left(X_{i}\right)\right)}\left(\mathcal{O}_{T^{1}\left(X_{i}\right)}, \mathcal{O}_{T^{1}\left(X_{i}\right)}\right) .
$$

Moreover, for any derived Artin stack $X, T^{1}(X)$ is affine over $X$ and can be written as a relative spectrum (see [HAG-II, Proposition 1.4.1.6])

$$
T^{1}(X) \simeq \operatorname{Spec}_{X}\left(\operatorname{Sym}\left(\mathbb{L}_{X}[-1]\right)\right) .
$$

In particular, we have natural quasi-isomorphisms

$$
\left.\underline{\mathbb{R} \operatorname{Hom}}\left(\mathcal{O}_{T^{1}(X)}, \mathcal{O}_{T^{1}(X)}\right) \simeq \underline{\mathbb{R} \underline{\operatorname{Hom}}}\left(\mathcal{O}_{X}, \bigoplus_{p} \wedge_{\mathcal{O}_{X}}^{p}\left(\mathbb{L}_{X}\right)[-p]\right)\right)
$$

We thus deduce that the natural morphism

$$
\underline{\mathbb{H o m}}_{L_{q c o h}(F)}\left(\mathcal{O}_{F}, \bigoplus_{p}\left(\wedge^{p} \mathbb{L}_{F / k}\right)[-p]\right) \longrightarrow \operatorname{Holim}_{i \in \Delta} \underline{\mathbb{H o m}}_{L_{q c o h}\left(X_{i}\right)}\left(\mathcal{O}_{X_{i}}, \bigoplus_{p}\left(\wedge^{p} \mathbb{L}_{X_{i} / k}\right)[-p]\right)
$$

is a quasi-isomorphism, which implies the lemma.

This finishes the proof of the proposition.

Remark 1.16 The interpretations of $p$-forms and closed $p$-forms as functions and invariant functions on the derived loop stacks given in 1.9 has a global counterpart. This globalization is not totally obvious, as the construction $X \mapsto \mathcal{L} X$ is not compatible with smooth gluing. However, it is possible to introduce the formal loop stack $\mathcal{L}^{f} X$, the formal completion of $\mathcal{L} X$ along the constant loops $X \hookrightarrow \mathcal{L} X$, and to prove that functions on $\mathcal{L}^{f} X$ have smooth descent (this is essentially the same argument as in our Lemma 1.15, see also Ben-Nad]). The group stack $\mathcal{H}$ acts on $\mathcal{L}^{f} X$, and if we denote by

$$
q:\left[\mathcal{L}^{f} X / \mathcal{H}\right] \longrightarrow B \mathcal{H} \longrightarrow B \mathbb{G}_{m}
$$

the natural projection, we have

$$
N C^{w}(F / k) \simeq q_{*}\left(\mathcal{O}_{\left[\mathcal{L}^{f} X / \mathcal{H}\right]}\right)
$$

In other words, closed $p$-forms on $X$ can be interpreted as $B \mathbb{G}_{a}$-invariant (or equivalently $S^{1}$-invariant) functions on $\mathcal{L}^{f} X$.

Remark 1.17 Let $F$ be a derived Artin stack locally of finite presentation over $k$. If we consider the absolute $\epsilon$-differential $D_{E}(\operatorname{Remark}$ [1.6) for $E:=\mathbf{D R}(F / k)[n-p]=$ $\mathbb{R} \Gamma\left(F, \oplus_{q \geq 0}\left(\wedge^{q} \mathbb{L}_{F}\right)[q]\right)[n-p]$, we get

$$
d_{D R}(p):=D_{E}(p): \operatorname{DR}(F / k)(p)[n-p] \longrightarrow \mathrm{NC}^{w}(F / k)(p+1)[n-(p+1)]
$$

whose geometric realization gives the derived de Rham differential (as a map of spaces)

$$
\mathrm{d}_{D R}(p):=\left|d_{D R}(p)\right|: \mathcal{A}^{p}(F ; n) \longrightarrow \mathcal{A}^{p+1, \mathrm{cl}}(F ; n) .
$$


We are now ready to define the space of $n$-shifted symplectic structures on a derived Artin stack $F$. To do this, let us recall that if $F$ is a derived Artin stack (locally of finite presentation over $k$, as are all of our derived Artin stacks by convention), then the cotangent complex $\mathbb{L}_{F / k}$ is a perfect complex. In particular it is a dualizable object in $L_{q c o h}(F)$ (or equivalently dualizable in $D_{q c o h}(F)$, see [To-Ve-1]). Its dual will be denoted by $\mathbb{T}_{F / k}$. Under this condition, any 2 -form $\omega$ of degree $n$ on $F$, induces by Proposition 1.14 a morphism of quasi-coherent complexes

$$
\mathcal{O}_{F} \longrightarrow\left(\wedge_{\mathcal{O}_{F}}^{2} \mathbb{L}_{F / k}\right)[n]
$$

and thus, by duality, a morphism $\wedge_{\mathcal{O}_{F}}^{2} \mathbb{T}_{F / k} \longrightarrow \mathcal{O}_{F}[n]$. By adjunction, this induces a well defined morphism in $L_{q c o h}(F)$

$$
\Theta_{\omega}: \mathbb{T}_{F / k} \longrightarrow \mathbb{L}_{F / k}[n]
$$

Definition 1.18 Let $F$ be a derived Artin stack, and $n \in \mathbb{Z}$.

1. A 2-form $\omega \in \mathcal{A}_{k}^{2}(F, n)$ is non-degenerate if the corresponding morphism in $D_{q c o h}(F)$

$$
\Theta_{\omega}: \mathbb{T}_{F / k} \longrightarrow \mathbb{L}_{F / k}[n]
$$

is an isomorphism.

We denote by $\mathcal{A}_{k}^{2}(F, n)^{n d}$ the full subspace of $\mathcal{A}_{k}^{2}(F, n)$ which is the union of all the connected components consisting of non-degenerate 2-forms of degree $n$ on $F$.

2. The space of $n$-shifted symplectic structures on $F$ (relative to $k$ ), $\operatorname{Symp}_{k}(F, n)$ is defined by the following homotopy pull-back square

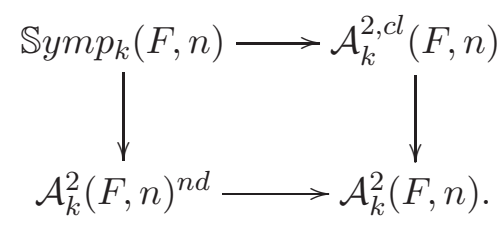

We will simply write $\operatorname{Symp}(F, n)$ when the base ring $k$ is clear from the context.

Note that, by definition, $\operatorname{Symp}(F, n)$ is the full sub-space of $\mathcal{A}^{2, c l}(F, n)$ defined by a unique condition on the underlying 2 -form.

Remark 1.19 As $F$ is locally of finite presentation, the cotangent complex $\mathbb{L}_{F / k}$ is perfect and thus of bounded amplitude. In particular we see that at most one of the spaces $\operatorname{Symp}(F, n)$ can be non-empty when $n$ varies in $\mathbb{Z}$, otherwise $\mathbb{L}_{F / k}$ will be periodic and thus not perfect.

More precisely, let us say that a derived Artin stack, locally of finite presentation over $k$, has amplitude in $[-m, n]$ (with $m, n \geq 0$ ) if its cotangent complex has perfect amplitude in that range. Then such a derived stack might only carry shifted symplectic structures of degree $r=m-n$. 
We conclude this section by describing the space of closed $p$-forms in two simple situations: smooth schemes (or more generally Deligne-Mumford stacks), and classifying stacks of reductive group schemes. Finally we show that shifted cotangent stacks carry a canonical shifted symplectic structure.

Smooth schemes. We start with the case of a smooth scheme $X$ over Speck. In this case $\mathbb{L}_{X / k} \simeq \Omega_{X / k}^{1}$, and thus Proposition 1.14 gives a description of the spaces of $p$-forms of degree $n$ as

$$
\mathcal{A}^{p}(X, n) \simeq\left|\mathbb{R} \Gamma\left(X, \Omega_{X / k}^{p}\right)[n]\right| .
$$

Assume first that $X=\operatorname{Spec} A$ is smooth and affine. In this case we know that $\mathbf{D R}(A / k)$ is naturally quasi-isomorphic, as a graded mixed complex, to $D R(A / k)$, the usual, underived, de Rham algebra of $A$ over $k$ (see [To-Ve-2]). We can then explicitly compute the graded complex $N C^{w}(A / k)$. By applying directly the definitions in Section 1.1. we get, for any $p \geq 0$

$$
N C^{w}(A / k)[-p](p) \simeq N C^{w}(D R(A / k))[-p](p) \simeq \Omega_{A / k}^{\geq p}
$$

where $\Omega_{A / k}^{\geq p}$ is the naively truncated de Rham complex

$$
\Omega_{A / k}^{p} \longrightarrow \Omega_{A / k}^{p+1} \longrightarrow \Omega_{A / k}^{p+2} \longrightarrow \ldots,
$$

where $\Omega_{A / k}^{p}$ sits in degree 0 . From this we deduce that the space of closed $p$-forms of degree $n$ on $\operatorname{Spec} A$ is

$$
\mathcal{A}^{p, c l}(\operatorname{Spec} A, n) \simeq\left|\Omega_{A / k}^{\geq p}[n]\right|
$$

In particular, we have

$$
\pi_{i}\left(\mathcal{A}^{p, c l}(\operatorname{Spec} A, n)\right) \simeq \begin{cases}0 & \text { if } n<0 \\ H_{D R}^{p+n-i}(\operatorname{Spec} A) & \text { if } 0 \leq i<n \\ \Omega_{A / k}^{p, c l} & \text { if } i=n\end{cases}
$$

By descent, we have similar formulas for a general smooth scheme $X$ (or more generally for a smooth Deligne-Mumford stack over $k$ )

$$
\begin{gathered}
\mathcal{A}^{p, c l}(X, n) \simeq\left|\mathbb{R} \Gamma\left(X, \Omega_{X / k}^{\geq p}\right)[n]\right|, \\
\pi_{i}\left(\mathcal{A}^{p, c l}(X, n)\right) \simeq H^{n-i}\left(X, \Omega_{X / k}^{\geq p}\right) .
\end{gathered}
$$

As a consequence, we have the following three important properties for a smooth scheme $X$

1. The space $\mathcal{A}^{p, c l}(X, 0)$ of closed $p$-forms of degree 0 is equivalent to the discrete set $\Gamma\left(X, \Omega_{X / k}^{p, c l}\right)$ of usual closed $p$-forms.

2. The spaces $\mathcal{A}^{p, c l}(X, n)$ are empty for $n<0$.

3. The spaces $\mathcal{A}^{p, c l}(X, n)$ are $n$-truncated for $n \geq 0$, and we have

$$
\pi_{i}\left(\mathcal{A}^{p, c l}(X, n)\right) \simeq H^{n-i}\left(X, \Omega_{X / k}^{\geq p}\right) .
$$


We can use these properties to describe $\operatorname{Symp}(X, n)$ for all smooth schemes $X$ and all $n$. Indeed, property (2) implies that $\operatorname{Symp}(X, n)$ is empty for all $n<0$. Property (1) gives that $\mathbb{S} y m p(X, 0)$ is equivalent to the set of usual symplectic forms on $X$. Finally, $\operatorname{Symp}(X, n)$ is also empty for $n>0$, as all closed 2-forms of degree $n$ must be degenerate, since we cannot have $\Omega_{X}^{1}[n] \simeq \mathrm{T}_{X}$ unless $n=0$ (note that both $\Omega_{X}^{1}$ and $\mathrm{T}_{X}$ are complexes concentrated in degree 0 since $X$ is smooth). In other words, on smooth schemes there are no derived symplectic forms of degree $\neq 0$, and those in degree 0 are just the usual ones.

Note also an interesting consequence of the above properties. When $X$ is smooth and also proper over Spec $k$, then we have

$$
\pi_{i}\left(\mathcal{A}^{p, c l}(X, n)\right) \simeq F^{p} H_{D R}^{n+p-i}(X / k),
$$

where $F^{*}$ stands for the Hodge filtration on the de Rham cohomology $H_{D R}^{*}(X / k)$.

Classifying stacks. Let now $G$ be an affine smooth group scheme over Speck, and consider its classifying stack $B G$, viewed as a derived Artin stack. If $\mathfrak{g}$ denotes the Lie algebra of $G$, the cotangent complex of $B G$ is $\mathfrak{g}^{\vee}[-1]$, considered as a quasi-coherent complex on $B G$ via the adjoint action of $G$ on its lie algebra $\mathfrak{g}$. In particular, using Proposition 1.14, we find that the spaces of $p$-forms on $B G$ are given by

$$
\mathcal{A}^{p}(B G, n) \simeq\left|\mathbb{H}\left(G, \operatorname{Sym}_{k}^{p}\left(\mathfrak{g}^{\vee}\right)\right)[n-p]\right|,
$$

where $\mathbb{H}(G,-)$ denotes the Hochschild cohomology complex of the affine group scheme $G$ with quasi-coherent coefficients (as in [De-Ga, §3.3] and [To3, §1.5]). In particular, when $G$ is reductive, we find

$$
\mathcal{A}^{p}(B G, n) \simeq\left|\operatorname{Sym}_{k}^{p}\left(\mathfrak{g}^{\vee}\right)^{G}[n-p]\right|,
$$

and, equivalently,

$$
\pi_{i}\left(\mathcal{A}^{p}(B G, n)\right) \simeq \begin{cases}0 & \text { if } i \neq n-p \\ \operatorname{Sym}_{k}^{p}\left(\mathfrak{g}^{\vee}\right)^{G} & \text { if } i=n-p\end{cases}
$$

Still under the hypothesis that $G$ is reductive, let us now compute the space $\mathcal{A}^{p, c l}(B G, n)$. For this we start by computing the graded mixed complex $\mathbf{D R}(B G)$. Proposition 1.14 tells us that the underlying graded complex of $\mathbf{D R}(B G)$ is cohomologically concentrated in degree 0

$$
\mathbf{D R}(B G) \simeq\left(S y m_{k}^{*} \mathfrak{g}^{\vee}\right)^{G}[0] .
$$

It follows that $\mathbf{D R}(B G)$, as a graded mixed complex is quasi-isomorphic to $\operatorname{Sym}_{k}^{*}\left(\mathfrak{g}^{\vee}\right)[0]$, where the $\epsilon$-action is trivial, and the grading is the natural grading on $S y m_{k}^{*}\left(\mathfrak{g}^{\vee}\right)$ (where $\mathfrak{g}^{\vee}$ is assigned weight 1). A direct consequence is that we have a natural quasi-isomorphism of graded complexes

$$
N C^{w}(\mathbf{D R}(B G)) \simeq \bigoplus_{i \geq 0} \operatorname{Sym}_{k}^{*}\left(\mathfrak{g}^{\vee}\right)^{G}[-2 i]
$$

Thus, we have

$$
\mathcal{A}^{p, c l}(B G, n) \simeq\left|\bigoplus_{i \geq 0} \operatorname{Sym}_{k}^{p+i}\left(\mathfrak{g}^{\vee}\right)^{G}[n-p-2 i]\right|
$$


In particular

$$
\pi_{0}\left(\mathcal{A}^{p, c l}(B G, n)\right) \simeq \begin{cases}0 & \text { if } n \text { is odd } \\ \operatorname{Sym}_{k}^{p}\left(\mathfrak{g}^{\vee}\right)^{G} & \text { if } n \text { is even }\end{cases}
$$

For degree reasons, a closed 2-form of degree $n$ can be non-degenerate on $B G$ only when $n=2$. Moreover,

$$
\pi_{0}\left(\mathcal{A}^{2, c l}(B G, 2)\right) \simeq \operatorname{Sym}_{k}^{2}\left(\mathfrak{g}^{\vee}\right)^{G},
$$

is the $k$-module of $G$-invariant symmetric bilinear forms on $\mathfrak{g}$. Such a closed 2-form is non-degenerate if and only if the corresponding bilinear form on $\mathfrak{g}^{\vee}$ is non-degenerate in the usual sense. As a consequence, we have

$$
\pi_{0}(\operatorname{Symp}(B G, 2)) \simeq\{\text { non-degenerate } G \text {-invariant quadratic forms on } \mathfrak{g}\} .
$$

If $G$ is assumed to have simple geometric fibers, then $\mathbb{S y m p}(B G, 2)$ possesses essentially a unique element. Indeed, $\operatorname{Sym}_{k}^{2}\left(\mathfrak{g}^{\vee}\right)^{G}$ is a projective module - being a direct factor in $\operatorname{Sym}_{k}^{2}\left(\mathfrak{g}^{\vee}\right)$. Moreover, it is well known that this projective module is of rank one when $k$ is a field, and this implies, by base change, that $S y m_{k}^{2}\left(\mathfrak{g}^{\vee}\right)^{G}$ is in fact a line bundle on Spec $k$. Nowhere vanishing sections of this line bundle corresponds to 2-shifted symplectic forms on $B G$. We thus see that, at least locally on the Zariski topology of Spec $k$, there is a 2-shifted symplectic form on $B G$, which is unique up to a multiplication by an invertible element of $k$.

When a reductive group scheme $G$ is realized as a closed subgroup scheme of $\mathrm{GL}_{n}$, then there is a natural element in $\operatorname{Symp}(B G, 2)$. The inclusion $G \hookrightarrow \mathrm{GL}_{n}$ defines a faithful representation $V$ of $G$ on $k^{n}$. This representation has a character, which is a $G$-invariant function on $G$. This function can be restricted to the formal completion of $G$ at the identity, to get a well defined element in

$$
\alpha_{V} \in \mathcal{O}\left(\widehat{G}^{G}\right) \simeq \widehat{\operatorname{Sym}}_{k}\left(\mathfrak{g}^{\vee}\right)^{G} .
$$

The degree 2 part of this element provides an invariant symmetric bilinear form on $\mathfrak{g}$, which is non-degenerate because $G$ is reductive, and thus a 2-shifted symplectic form on $B G$. In different terms, $B \mathrm{GL}_{n}$ has a canonical 2-shifted symplectic structure, given by the bilinear form $(A, B) \mapsto \operatorname{Tr}(A B)$, defined on the $k$-modules $\mathcal{M}_{n}(k)$ of $n \times n$ matrices. The inclusion $G \hookrightarrow \mathrm{GL}_{n}$ defines a morphism of stacks $B G \longrightarrow B \mathrm{GL}_{n}$, and the pull-back of the canonical 2-shifted symplectic form on $B G$ remains a 2-shifted symplectic form.

Shifted cotangent stacks. We define the $n$-shifted cotangent stack of a DeligneMumford stack, and prove that it carries a canonical $n$-shifted symplectic structure.

Definition 1.20 Let $n \in \mathbb{Z}$, and $X$ be a derived Artin stack locally of finite presentation over $k$. We define the $n$-shifted cotangent (derived) stack as

$$
\mathrm{T}^{*} X[n]:=\mathbb{R S p e c} \operatorname{Sym}_{\mathcal{O}_{X}}\left(\mathbb{T}_{X}[-n]\right) .
$$

For any $n$, we have a map of derived stacks $p[n]: \mathrm{T}^{*} X[n] \longrightarrow X$, induced by the canonical $\operatorname{map} \mathcal{O}_{X} \longrightarrow \operatorname{Sym}_{\mathcal{O}_{X}}\left(\mathbb{T}_{X}[-n]\right)$. 
On $\mathrm{T}^{*} X[n]$ we have a canonical Liouville $n$-shifted 1 -form. The idea - like in differential geometry, where there is obviously no shift - is that the pullback of $p[n]: T^{*} X[n] \rightarrow$ $X$ along $p[n]$ itself has a canonical section, the diagonal, and any such section gives a horizontal 1-form on $T^{*} X[n]$.

More precisely, we start by considering the inclusion

$$
\mathbb{T}_{X}[-n] \hookrightarrow \operatorname{Sym}_{\mathcal{O}_{X}}\left(\mathbb{T}_{X}[-n]\right) .
$$

Since $\mathbb{T}_{X}$ is perfect, this corresponds uniquely - by adjunction - to a map

$\mathcal{O}_{X} \rightarrow \mathbb{T}_{X}[-n]^{\vee} \otimes_{\mathcal{O}_{X}} \operatorname{Sym}_{\mathcal{O}_{X}}\left(\mathbb{T}_{X}[-n]\right) \simeq \mathbb{L}_{X}[n] \otimes_{\mathcal{O}_{X}} \operatorname{Sym}_{\mathcal{O}_{X}}\left(\mathbb{T}_{X}[-n]\right) \simeq p[n]_{*} p[n]^{*} \mathbb{L}_{X}[n]$,

that, again by adjution, yields a map

$$
\mathcal{O}_{T^{*} X[n]} \rightarrow p[n]^{*} \mathbb{L}_{X}[n] .
$$

By composing this arrow with the shift-by- $n$ of the canonical map $p[n]^{*} \mathbb{L}_{X} \rightarrow \mathbb{L}_{T^{*} X[n]}$, we obtain the Liouville n-shifted 1 -form on $\mathrm{T}^{*} X[n]$

$$
\lambda(X ; n): \mathcal{O}_{T^{*} X[n]} \rightarrow \mathbb{L}_{T^{*} X[n]}[n] .
$$

Nothe that $\lambda$ is horizontal, by definition, i.e. the composite $\mathcal{O}_{T^{*} X[n]} \rightarrow \mathbb{L}_{T^{*} X[n]}[n] \rightarrow$ $\mathbb{L}_{T^{*} X[n] / X}[n]$ is zero.

Recall from Remark 1.17, the existence of a derived de Rham differential

$$
\mathrm{d}_{D R}:=\left|d_{D R}(1)\right|: \mathcal{A}^{1}\left(T^{*} X[n] ; n\right) \longrightarrow \mathcal{A}^{2, \mathrm{cl}}\left(T^{*} X[n] ; n\right) .
$$

Let's denote by $\mathrm{d}_{D R}(\lambda(X ; n)) \in \pi_{0}\left(\mathcal{A}^{2, \mathrm{cl}}\left(T^{*} X[n] ; n\right)\right)=H^{n-2}\left(N C^{w}\left(T^{*} X[n]\right)(2)\right)$ the induced $n$-shifted closed 2 -form.

Proposition 1.21 If $X$ is a derived Deligne-Mumford stack, then the underlying $n$-shifted 2 -form of $\omega:=d_{D R}(\lambda(X ; n))$ is non degenerate, i.e. $\omega$ is symplectic.

Proof. Let us simply denote by $d \lambda^{b}: \mathbb{T}_{\mathrm{T}^{*} X[n]} \rightarrow \mathbb{L}_{\mathrm{T}^{*} X[n]}[n]$ the map induced - by adjunction - by the 2 -form in $H^{n}\left(T_{X}^{*}[n], \wedge^{2} \mathbb{L}_{\mathrm{T}^{*} X[n]}\right)$ underlying $\mathrm{d}_{D R}(\lambda(X ; n))$. We want to prove that $d \lambda^{b}$ is a quasi-isomorphism. Since étale maps induce equivalences of cotangent complexes, by using an étale atlas $\left\{U_{i}=\operatorname{Spec}\left(A_{i}\right) \rightarrow X\right\}$ for $X$, and the induced étale atlas $\left\{\mathrm{T}^{*} U_{i}[n]=\operatorname{Spec}\left(B_{i}\right) \rightarrow \mathrm{T}^{*} X[n]\right\}$ on $\mathrm{T}^{*} X[n]$, it will suffice to prove the same statement upon restriction along any such étale map $\operatorname{Spec}\left(B_{i}\right) \rightarrow \mathrm{T}^{*} X[n]$. By naturality of the construction of $d \lambda^{b}$ with respect to étale maps, it will be enough to prove the proposition for $X=\operatorname{Spec}(A)$ (with $A$ quasi-free).

So, let $X=\operatorname{Spec}(A)$, where $A$ is a quasi-free $k$-cdga on the quasi-basis $\left\{x_{i}\right\}_{i \in I}$. Then

$$
\mathbb{L}_{X} \simeq \Omega_{A / k}^{1}=\oplus_{i \in I} A \delta x_{i}
$$

with $\left|\delta x_{i}\right|=\left|x_{i}\right|$, with the usual differential defined by $d\left(a \delta x_{i}\right)=d(a) \delta x_{i}+(-1)^{|a|} a \delta\left(d x_{i}\right)$, where $\delta$ is the unique derivation $A \rightarrow \Omega_{A / k}^{1}$ extending $x_{i} \mapsto \delta x_{i}$. Therefore,

$$
\mathbb{T}_{X} \simeq \mathrm{T}_{A / k}=\oplus_{i \in I} A \xi_{i}
$$


where $\xi_{i}$ is dual to $\delta x_{i}$, and $\left|\xi_{i}\right|=-\left|x_{i}\right|$. Moreover

$$
Y:=T^{*} X[n]=\operatorname{Spec}\left(\operatorname{Sym}_{\mathrm{A}}\left(\mathbb{T}_{X}[-n]\right)\right) \simeq \operatorname{Spec}(B),
$$

where $B$ is quasi-free over $A$ with quasi-basis $\left\{y_{i}\right\}_{i \in I},\left|y_{i}\right|=-\left|x_{i}\right|+n$. In other words, $B$ is quasi-free over $k$ with quasi-basis $\left\{x_{i}, y_{i}\right\}_{i \in I},\left|y_{i}\right|=-\left|x_{i}\right|+n$. Therefore

$$
\mathbb{L}_{Y} \simeq \Omega_{B / k}^{1}=\oplus_{i \in I} B \delta x_{i} \oplus B \delta y_{i},
$$

with $\left|\delta x_{i}\right|=\left|x_{i}\right|,\left|\delta y_{i}\right|=-\left|x_{i}\right|+n$ (with its usual differential), and

$$
\mathbb{T}_{Y} \simeq \mathrm{T}_{B / k}=\oplus_{i \in I} B \xi_{i} \oplus B \eta_{i},
$$

where $\xi_{i}$ is dual to $\delta x_{i}, \eta_{i}$ is dual to $\delta y_{i}$, and $\left|\xi_{i}\right|=-\left|x_{i}\right|,\left|\eta_{i}\right|=\left|x_{i}\right|-n$. Moreover

$$
\mathbb{L}_{Y / X} \simeq \Omega_{B / A}^{1}=\oplus_{i \in I} B \delta y_{i}, \quad \mathbb{T}_{Y / X} \simeq \mathrm{T}_{B / A}=\oplus_{i \in I} B \eta_{i}
$$

In these terms, the $n$-shifted Liouville 1 -form $\lambda$ on $Y$ is given by $\lambda=\sum_{i \in I}(-1)^{\left|y_{i}\right|} y_{i} \delta x_{i}$. Note that $\lambda$ is an element of degree 0 in $D R(Y / k)(1)[n-1] \simeq \Omega_{B / k}^{1}[n]$. Now, by definition of the de Rham differential (Remark 1.17), we have

$d_{D R} \lambda=\left(d_{D R}\left(\sum_{i \in I}(-1)^{\left|y_{i}\right|} y_{i} \delta x_{i}\right)=\sum_{i \in I}(-1)^{\left|y_{i}\right|} \delta y_{i} \wedge \delta x_{i}, 0, \ldots, 0, \ldots\right) \in\left(N C^{w}(D R(Y / k))(2)[n-2]\right)^{0}$,

so that the $n$-shifted 2 -form underlying $d_{D R} \lambda$ is $\sum_{i \in I}(-1)^{\left|y_{i}\right|} \delta y_{i} \wedge \delta x_{i}$. In particular, we have

$$
\begin{gathered}
d \lambda^{b}: \mathrm{T}_{B / k}=\oplus_{i \in I} B \xi_{i} \oplus B \eta_{i} \longrightarrow \oplus_{i \in I} B \delta x_{i}[n] \oplus B \delta y_{i}[n]=\Omega_{B / k}^{1}[n], \\
\xi_{i} \longmapsto-(-1)^{\left|y_{i}\right|} \delta y_{i}[n], \quad \eta_{i} \longmapsto(-1)^{\left|y_{i}\right|} \delta x_{i}[n],
\end{gathered}
$$

and this is, by inspection, an isomorphism of graded $B$-modules, and we conclude that $\omega$ is indeed symplectic (since we already knew that $d \lambda^{b}$ is a map of $B$-dg-modules).

Remark 1.22 It is very likely that Proposition 1.21 holds for any derived Artin stack, we just did not investigate how one may deal with smooth atlases instead of étale ones.

\section{Existence of shifted symplectic structures}

We prove in this section three existence results for $n$-shifted symplectic forms on certain derived stacks. We start by the mapping derived stack of an oriented object to an $n$ shifted symplectic target, which is surely the most important of the existence theorems given below. We also introduce the notion of a Lagrangian structure on a morphism with target an $n$-shifted symplectic derived stack, and show that the fibered product of two such morphisms comes equipped with a natural $(n-1)$-shifted symplectic form. Finally, we explain a construction of 2-shifted symplectic forms using the Chern character construction of [To-Ve-1], and apply it to exhibit a natural 2-shifted symplectic structure on $\mathbb{R}$ Perf, the derived moduli stack of perfect complexes. 


\subsection{Mapping stacks}

The following construction is well-known in differential geometry and provides, together with [AKSZ, heuristics for our constructions below. Let $M$ be a compact $C^{\infty}$-manifold of dimension $m, N$ be a $C^{\infty}$-manifold, and $\operatorname{Map}_{C^{\infty}}(M, N)$ the Fréchet manifold of $C^{\infty}$-maps from $M$ to $N$. The following diagram

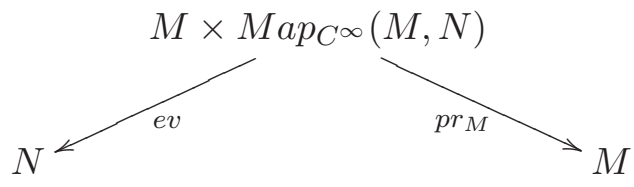

induces a map

$$
\Omega_{M}^{p} \times \Omega_{N}^{q} \rightarrow \Omega_{M a p_{C}(M, N)}^{p+q-m}:(\alpha, \beta) \mapsto \int_{M} p r_{M}^{*} \alpha \wedge e v^{*} \beta:=\widehat{\alpha \beta},
$$

where $\int_{M}$ denotes integration along the fiber, sometimes called the hat-product (see e.g. Viz]). Now, if $(N, \omega)$ is symplectic, then $\widehat{1 \omega} \in \Omega_{M a p(M, N)}^{2-m}$ defines a symplectic form on $\operatorname{Map}_{C \infty}(M, N)$.

In the derived algebraic geometry setting we are concerned with in this paper, we will need a replacement for Poincaré duality and for the notion of an orientation. The first one is given by Serre duality (in the more general context of Calabi-Yau categories), while the second one will be that of $\mathcal{O}$-orientation (Definition 2.4 . The notion of $\mathcal{O}$-orientation will allow for a quasi-coherent variant of integration along the fiber (Definition 2.3).

We start by considering the following finiteness conditions on derived stacks.

Definition 2.1 A derived stack $X$, over a derived affine scheme $\mathbf{S p e c} A$, is strictly $\mathcal{O}_{-}$ compact over $A$ if it satisfies the following two conditions

1. $\mathcal{O}_{X}$ is a compact object in $D_{q c o h}(X)$.

2. For any perfect complex $E$ on $X$, the $A$-dg-module

$$
C(X, E):=\mathbb{R} \underline{\operatorname{Hom}}\left(\mathcal{O}_{X}, E\right)
$$

is perfect.

$A$ derived stack $X$ over $k$ is $\mathcal{O}$-compact if for any derived affine scheme $\mathbf{S p e c} A$ the derived stack $X \times \operatorname{Spec} A$ is strictly $\mathcal{O}$-compact over $A$.

Remark 2.2 Since perfect complexes are exactly the dualizable objects in $D_{q c o h}(X)$, condition (1) of the definition above implies that all perfect complexes are compact in $D_{q c o h}(X)$ for an $\mathcal{O}$-compact derived stack $X$, as well as all perfect complexes on $X \times \operatorname{Spec} A$ for any $A \in \operatorname{cdga}_{k}^{\leq 0}$.

The main property of (strictly) $\mathcal{O}$-compact derived stacks is the existence, for any other derived stack $F$, of a morphism of graded mixed complexes (over $k$ )

$$
\kappa_{F, X}: \mathbf{D R}(F \times X) \longrightarrow \mathbf{D R}(F) \otimes_{k} C\left(X, \mathcal{O}_{X}\right)
$$


functorial in $F$, where $C\left(X, \mathcal{O}_{X}\right)$ is considered pure of weight 0 with trivial mixed structure. It is defined as follows.

Since $X$ is, by hypothesis, $\mathcal{O}$-compact, the complex $C\left(X, \mathcal{O}_{X}\right)$ is perfect over $k$, so the $\infty$-endofunctor $E \mapsto E \otimes_{k} C\left(X, \mathcal{O}_{X}\right)$, of the $\infty$-category of mixed graded complexes, commutes with $\infty$-limits. Also, by definition, the functor $F \mapsto \mathbf{D R}(F \times X)$ sends $\infty$ colimits to $\infty$-limits. Therefore, the two $\infty$-functors

$$
\mathbf{D R}(-\times X), \mathbf{D R}(-) \otimes_{k} C\left(X, \mathcal{O}_{X}\right): \mathbf{d} \mathbf{S t}_{k}^{o p} \longrightarrow \epsilon-\mathbf{d g}_{k}^{g r}
$$

send $\infty$-colimits of derived stacks to $\infty$-limits of graded complexes. By left Kan extensions (see [Lu1] or [To-Ve-1, §1.2]), we see that in order to construct a natural transformation $\kappa_{-, X}: \mathbf{D R}(-\times X) \longrightarrow \mathbf{D R}(-) \otimes_{k} C\left(X, \mathcal{O}_{X}\right)$, it is enough to construct a natural transformation between the two $\infty$-functors restricted to the $\infty$-category of derived affine schemes. By definition, these two $\infty$-functors, restricted to derived affine schemes are given as follows

$$
\begin{aligned}
& \mathbf{D R}(-) \otimes_{k} C\left(X, \mathcal{O}_{X}\right): \quad \mathbf{d A f f}_{k}^{o p} \equiv \operatorname{cdga}_{k}^{\leq 0} \quad \longrightarrow \quad \epsilon-\mathbf{d g}_{k}^{g r} \\
& A \quad \longmapsto \mathbf{D R}(A) \otimes_{k} C\left(X, \mathcal{O}_{X}\right) \\
& \mathbf{D R}(-\times X): \mathbf{d A f f}_{k}^{o p} \equiv \mathbf{c d g a}_{k}^{\leq 0} \longrightarrow \quad \longrightarrow-\mathbf{d g}_{k}^{g r} \\
& A \quad \longmapsto H\left(X, \mathbf{D R}\left(A \otimes_{k} \mathcal{O}_{X}\right)\right) \text {, }
\end{aligned}
$$

where $\mathbf{D R}\left(A \otimes_{k} \mathcal{O}_{X}\right)$ is the stack of mixed graded complexes on $X$ sending $\operatorname{Spec} B \rightarrow X$ to $\mathbf{D R}\left(A \otimes_{k} B\right)$, and $H\left(X, \mathbf{D R}\left(A \otimes_{k} \mathcal{O}_{X}\right)\right)$ denotes its global sections.

For any two objects $B, C \in \mathbf{c d g a}^{\leq 0}$, we have a natural equivalence of graded mixed complexes (Kunneth formula)

$$
\mathbf{D R}(B) \otimes_{k} \mathbf{D R}(C) \simeq \mathbf{D R}\left(B \otimes_{k} C\right)
$$

induced by the identification

$$
\mathbb{L}_{B \otimes_{k} C} \simeq\left(\mathbb{L}_{B} \otimes_{k} C\right) \oplus\left(B \otimes_{k} \mathbb{L}_{C}\right) .
$$

Therefore, the $\infty$-functor $\mathbf{D R}(-\times X)$ sends $A$ to $H\left(X, \mathbf{D R}(A) \otimes_{k} \mathbf{D R}\left(\mathcal{O}_{X}\right)\right)$. We consider the natural projection on the component of weight zero (with trivial mixed structure) $\mathbf{D R}\left(\mathcal{O}_{X}\right) \longrightarrow \mathcal{O}_{X}$, and obtain a morphism $\mathbf{D R}(-\times X) \longrightarrow H\left(X, \mathbf{D R}(-) \otimes_{k} \mathcal{O}_{X}\right) \simeq$ $C\left(X, \mathbf{D R}(-) \otimes_{k} \mathcal{O}_{X}\right)$. As $X$ is $\mathcal{O}$-compact, $C(X,-)$ commutes with colimits of quasicoherent sheaves, and thus the natural morphism

$$
\mathbf{D R}(-) \otimes_{k} C\left(X, \mathcal{O}_{X}\right) \longrightarrow C\left(X, \mathbf{D R}(-) \otimes_{k} \mathcal{O}_{X}\right)
$$

is an equivalence.

We thus have defined a natural transformation of $\infty$-functors

$$
\mathbf{D R}(-\times X) \longrightarrow C\left(X, \mathbf{D R}(-) \otimes_{k} \mathcal{O}_{X}\right) \simeq \mathbf{D R}(-) \otimes_{k} C\left(X, \mathcal{O}_{X}\right),
$$

which defines our morphism of graded mixted complexes

$$
\kappa_{F, X}: \mathbf{D R}(F \times X) \longrightarrow \mathbf{D R}(F) \otimes_{k} C\left(X, \mathcal{O}_{X}\right) .
$$


We can apply the $\infty$-functor $N C^{w}$ to the morphism above, in order to get a morphism of graded complexes

$$
\kappa_{F, X}: N C^{w}(F \times X) \longrightarrow N C^{w}\left(\mathbf{D R}(F) \otimes_{k} C\left(X, \mathcal{O}_{X}\right)\right) .
$$

As $C\left(X, \mathcal{O}_{X}\right)$ is a perfect complex over $k$, the morphism

$$
N C^{w}(\mathbf{D R}(F)) \otimes_{k} C\left(X, \mathcal{O}_{X}\right) \longrightarrow N C^{w}\left(\mathbf{D R}(F) \otimes_{k} C\left(X, \mathcal{O}_{X}\right)\right)
$$

is an equivalence of graded complexes.

To summarized, we have defined for any derived stack $F$ and any $\mathcal{O}$-compact derived stack $X$, a commutative square of graded complexes

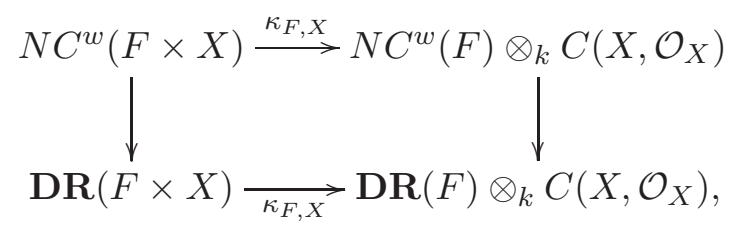

where the vertical morphisms are the projections $N C^{w} \longrightarrow \mathbf{D R}$.

We keep the hypothesis that $X$ is an $\mathcal{O}$-compact derived stack, and we assume further that, for some integer $d \in \mathbb{Z}$, we are given a map

$$
\eta: C\left(X, \mathcal{O}_{X}\right) \longrightarrow k[d]
$$

in the derived category $D(k)$. Then, for any derived stack $F$ we have a natural morphism of graded complexes

$$
N C^{w}(F \times X) \stackrel{\kappa_{F, X}}{\longrightarrow} N C^{w}(F) \otimes_{k} C\left(X, \mathcal{O}_{X}\right) \stackrel{i d \otimes \eta}{\longrightarrow} N C^{w}(F)[d] .
$$

This morphism, well defined in the homotopy category of graded complexes $\operatorname{Ho}\left(\mathbf{d g}_{k}^{g r}\right)$, is called the integration along $\eta$.

Definition 2.3 Let $F$ and $X$ be derived stacks, with $X$ O-compact, and let $\eta: C\left(X, \mathcal{O}_{X}\right) \longrightarrow k[d]$ be a morphism in $D(k)$, for some integer $d$. The integration map along $\eta$ is the morphism

$$
\int_{\eta}: N C^{w}(F \times X) \stackrel{\kappa_{F, X}}{\longrightarrow} N C^{w}(F) \otimes_{k} C\left(X, \mathcal{O}_{X}\right) \stackrel{i d \otimes \eta}{\longrightarrow} N C^{w}(F)[d]
$$

constructed above.

We also have a similar morphism on the level of de Rham complexes

$$
\int_{\eta}: \mathbf{D R}(F \times X) \stackrel{\kappa_{F, X}}{\longrightarrow} \mathbf{D R} \otimes_{k} C\left(X, \mathcal{O}_{X}\right) \stackrel{i d \otimes \eta}{\longrightarrow} \mathbf{D R}(F)[d],
$$

in a way that we have a commutative diagram of mixed graded complexes

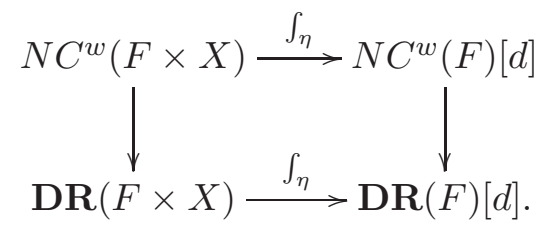


Let $X$ be an $\mathcal{O}$-compact derived stack. The complex $C\left(X, \mathcal{O}_{X}\right)$ possesses a natural structure of a (commutative) dg-algebra over $k$, so comes equipped with a cup-product morphism

$$
C\left(X, \mathcal{O}_{X}\right) \otimes_{k} C\left(X, \mathcal{O}_{X}\right) \longrightarrow C\left(X, \mathcal{O}_{X}\right)
$$

In particular, any morphism $\eta: C\left(X, \mathcal{O}_{X}\right) \longrightarrow k[-d]$ provides a morphism

$$
-\cap \eta: C\left(X, \mathcal{O}_{X}\right) \otimes C\left(X, \mathcal{O}_{X}\right) \stackrel{\cap}{\longrightarrow} C\left(X, \mathcal{O}_{X}\right) \stackrel{\eta}{\longrightarrow} k[-d]
$$

If we denote by $C\left(X, \mathcal{O}_{X}\right)^{\vee}:=\mathbb{R} \underline{\operatorname{Hom}}\left(C\left(X, \mathcal{O}_{X}\right), k\right)$ the derived dual of $C\left(X, \mathcal{O}_{X}\right)$, the morphism above defines an adjoint morphism

$$
-\cap \eta: C\left(X, \mathcal{O}_{X}\right) \longrightarrow C\left(X, \mathcal{O}_{X}\right)^{\vee}[-d]
$$

More generally, if $E$ is a perfect complex on $X$, of dual $E^{\vee}:=\mathbb{R} \underline{\mathcal{H} o m}\left(E, \mathcal{O}_{X}\right)$, the natural pairing

$$
C(X, E) \otimes_{k} C\left(X, E^{\vee}\right) \longrightarrow C\left(X, \mathcal{O}_{X}\right),
$$

composed with $\eta$ induces a morphism

$$
-\cap \eta: C(X, E) \longrightarrow C\left(X, E^{\vee}\right)^{\vee}[-d] .
$$

If moreover $A \in \mathbf{c d g a}_{k}^{\leq 0}$, the same is true for the derived $A$-scheme $X_{A}:=X \times \operatorname{Spec} A$. The morphism $\eta$ induces a morphism

$$
\eta_{A}=\eta \otimes i d_{A}: C\left(X_{A}, \mathcal{O}_{X_{A}}\right) \simeq C\left(X, \mathcal{O}_{X}\right) \otimes_{k} A \longrightarrow k[-d] \otimes_{k} A,
$$

and for any perfect complex $E$ on $X_{A}$, the morphism $\eta_{A}$ induces a natural morphism

$$
-\cap \eta_{A}: C\left(X_{A}, E\right) \longrightarrow C\left(X_{A}, E^{\vee}\right)^{\vee}[-d]
$$

where now $C\left(X_{A}, E^{\vee}\right)^{\vee}$ is the derived $A$-dual of $C\left(X_{A}, E^{\vee}\right)$.

Definition 2.4 Let $X$ be an $\mathcal{O}$-compact derived stack and $d \in \mathbb{Z}$. An $\mathcal{O}$-orientation of degree $d$ on $X$ consists of a morphism of complexes

$$
[X]: C\left(X, \mathcal{O}_{X}\right) \longrightarrow k[-d],
$$

such that for any $A \in \mathbf{c d g a}_{k}^{\leq 0}$ and any perfect complex $E$ on $X_{A}:=X \times \operatorname{Spec} A$, the morphism

$$
-\cap[X]_{A}: C\left(X_{A}, E\right) \longrightarrow C\left(X_{A}, E^{\vee}\right)^{\vee}[-d]
$$

is a quasi-isomorphism of $A$-dg-modules.

We are now ready to state and prove our main existence statement.

Theorem 2.5 Let $F$ be a derived Artin stack equipped with an $n$-shifted symplectic form $\omega \in \mathbb{S} y m p(F, n)$. Let $X$ be an $\mathcal{O}$-compact derived stack equipped with an $\mathcal{O}$-orientation $[X]: C\left(X, \mathcal{O}_{X}\right) \longrightarrow k[-d]$ of degree d. Assume that the derived mapping stack $\operatorname{Map}(X, F)$ is itself a derived Artin stack locally of finite presentation over $k$. Then, $\operatorname{Map}(X, F)$ carries a canonical $(n-d)$-shifted symplectic structure. 
Proof. We let $\pi: X \times \operatorname{Map}(X, F) \longrightarrow F$ be the evaluation morphism. We have $\omega \in \mathbb{S} y m p(F, n) \subset \mathcal{A}^{2, c l}(F, n)$, and this corresponds to a morphism of graded complexes

$$
\omega: k[2-n](2) \longrightarrow N C^{w}(F) .
$$

Using the integration along the orientation $[X]$ of definition 2.3 , we consider the composition

$$
\int_{[X]} \omega: k[2-n](2) \stackrel{\omega}{\longrightarrow} N C^{w}(F) \stackrel{\pi^{*}}{\longrightarrow} N C^{w}(X \times \operatorname{Map}(X, F)) \stackrel{\int_{[X]}}{\longrightarrow} N C^{w}(\operatorname{Map}(X, F))[-d] .
$$

This is, by definition, a closed 2 -form of degree $(n-d)$ on $\operatorname{Map}(X, F)$. i.e.

$$
\int_{[X]} \omega \in \mathcal{A}^{2, c l}(\operatorname{Map}(X, F), n-d) .
$$

It remains to show that this 2 -form is non-degenerate. For this, we have to determine the underlying 2 -form of degree $(n-d)$. It is given by the following morphism

$$
k[2-n](2) \stackrel{\omega}{\longrightarrow} \mathbf{D R}(F) \stackrel{\pi^{*}}{\longrightarrow} \mathbf{D R}(X \times \operatorname{Map}(X, F)) \stackrel{\int_{[X]}^{\longrightarrow}}{\longrightarrow} \mathbf{D R}(\operatorname{Map}(X, F))[-d] .
$$

If we unravel the definition of $\int_{[X]}$, we see that this 2-form can be described as follows.

First of all, let $x: \operatorname{Spec} A \longrightarrow \operatorname{Map}(X, F)$ be an $A$-point corresponding to a morphism of derived stacks

$$
f: X \times \operatorname{Spec} A \longrightarrow F \text {. }
$$

If $\mathbb{T}_{F}$ denotes the tangent complex of $F$, the tangent complex of $\operatorname{Map}(X, F)$ at the point $x$ is given by

$$
\mathbb{T}_{x} \operatorname{Map}(X, F) \simeq C\left(X_{A}, f^{*}\left(\mathbb{T}_{F}\right)\right) .
$$

The 2 -form $\omega$ defines a non-degenerate pairing of perfect complexes on $F$

$$
\mathbb{T}_{F} \wedge \mathbb{T}_{F} \longrightarrow \mathcal{O}_{F}[n]
$$

which induces an alternate pairing of $A$-dg-modules

$$
C\left(X_{A}, f^{*}\left(\mathbb{T}_{F}\right)\right) \wedge C\left(X_{A}, f^{*}\left(\mathbb{T}_{F}\right)\right) \longrightarrow C\left(X_{A}, \mathcal{O}_{X_{A}}[n]\right) .
$$

We can compose with the orientation $\left[X_{A}\right]$ to get a pairing of perfect $A$-dg-modules.

$$
C\left(X \times \operatorname{Spec} A, f^{*}\left(\mathbb{T}_{F}\right)\right) \wedge C\left(X \times \operatorname{Spec} A, f^{*}\left(\mathbb{T}_{F}\right)\right) \longrightarrow A[n-d] .
$$

By inspection, this pairing is the one induced by the 2-form underlying $\int_{[X]} \eta$. The fact that it is non-degenerate then follows from the definition of an orientation and the fact that $\omega$ is non-degenerate.

Here follow some examples of derived stacks $X$ satisfying the condition of the theorem 2.5 , 
- Mapping stacks with Betti source. Let $M$ be a compact, connected, and oriented topological manifold. We consider $X=S(M)$ its simplicial set of singular simplices, as a constant derived stack. The category $D_{q c o h}(X)$ is then naturally equivalent to $D_{l o c}(M, k)$, the full sub-category of the derived category of sheaves of $k$-modules on the space $M$, consisting of objects with locally constant cohomology sheaves. In particular, we have functorial isomorphisms

$$
H^{*}(X, E) \simeq H^{*}(M, \mathcal{E})
$$

for any $E \in D_{q c o h}(X)$ whose corresponding complex of $k$-modules on $M$ is denoted by $\mathcal{E}$. Perfect complexes on $X$ correspond to objects in $D_{l o c}(M, k)$ locally quasi-isomorphic to bounded complexes of constant sheaves of projective modules of finite type. This implies that $X$ is $\mathcal{O}$-compact. Moreover, the orientation on $M$ determines a well defined fundamental class $[M] \in H_{d}(M, k)$, and thus a morphism $[X]: C(M, k) \longrightarrow k[-d]$, where $d=\operatorname{dim} M$. Poincaré duality on $M$ implies that $[X]$ is an $\mathcal{O}$-orientation on $X$. Finally, $M$ has the homotopy type of a finite $\mathrm{CW}$ complex, so $X$ is a finite homotopy type. This implies that for any derived Artin stack $F, \operatorname{Map}(X, F)$ is a finite homotopy limit of copies of $F$, and thus is itself a derived Artin stack.

- Mapping stacks with de Rham source. Let $Y$ be a smooth and proper DeligneMumford stack over Spec $k$, with connected geometric fibers. Recall from HAG-II, Corollary 2.2.6.15] that we can define a derived stack $Y_{D R}$, such that

$$
Y_{D R}(A):=Y\left(\pi_{0}(A)_{r e d}\right)
$$

for any $A \in \operatorname{cdga}_{k}^{\leq 0}$. We set $X:=Y_{D R}$. We have a natural equivalence between $D_{q c o h}(X)$ and the derived category of $\mathcal{D}_{Y / k}$-modules with quasi-coherent cohomology. Moreover, perfect complexes on $X$ correspond to complexes of $\mathcal{D}_{Y / k}$-modules whose underlying quasi-coherent complexes are perfect over $Y$. In particular, if $E$ is a perfect complex on $X$, corresponding to a complex of $\mathcal{D}_{Y / k}$-modules $\mathcal{E}$ perfect over $Y$, then we have

$$
H^{*}(X, E) \simeq H_{D R}^{*}(Y / k, \mathcal{E}) .
$$

It follows easily that $X$ is $\mathcal{O}$-compact. Moreover, the choice of a fundamental class in de Rham cohomology $[Y] \in H_{D R}^{2 d}(Y / k, \mathcal{O})$ (where $d$ is the relative dimension of $Y$ over Spec $k$ ) determines a morphism

$$
[X]: C(X, \mathcal{O}) \longrightarrow k[-2 d]
$$

which, by Poincaré duality in de Rham cohomology, is an $\mathcal{O}$-orientation on $X$.

Finally, the fact that $\operatorname{Map}(X, F)$ is a derived Artin stack when $F$ is one, can be deduced from Lurie's version of Artin representability criterion. We will be mainly interested in the special case where $F$ is either a smooth quasi-projective variety, or a classifying stack $B G$, or the derived stack of perfect complexes $\mathbb{R}$ Perf. In all these specific situations, the fact that $\operatorname{Map}(X, F)$ is a derived Artin stack locally of finite presentation can be found in [HAG-II, $§ 2.2 .6 .3$ ] and [Si2]. 
- Mapping stacks with Dolbeault source. The previous example has the following Dolbeault analog. Let again $Y$ be a smooth and proper Deligne-Mumford stack over Spec $k$, with connected geometric fibers. We define $Y_{D o l}$ by (see [Si2, §2])

$$
Y_{D o l}:=B \widehat{\mathbb{T}}_{Y / k} \longrightarrow Y,
$$

the classifying stack of the formal tangent bundle of $Y$ relative to $k$. We set $X:=$ $Y_{D o l}$. We know that a quasi-coherent complex $E$ on $X$ consists of a pair $(\mathcal{E}, \phi)$ where $\mathcal{E}$ is a quasi-coherent complex on $Y$, and $\phi$ is a Higgs field $\phi$ on $\mathcal{E}$ (i.e. an action of the $\mathcal{O}_{Y}$-algebra $\left.\operatorname{Sym}_{\mathcal{O}_{Y}}\left(\mathbb{T}_{Y / k}\right)\right)$. Under this correspondence, we have

$$
H^{*}(X, E) \simeq H_{D o l}^{*}(Y, \mathcal{E}) .
$$

This implies that $X$ is $\mathcal{O}$-compact. As above, the choice of a fundamental class in Hodge cohomology $[Y] \in H_{D o l}^{2 d}(Y, \mathcal{O}) \simeq H^{d}\left(Y, \Omega_{Y / k}^{d}\right)$ determines a morphism

$$
[X]: C(X, \mathcal{O}) \longrightarrow k[-2 d]
$$

which, by Poincaré duality in Dolbeault cohomology, is indeed an $\mathcal{O}$-orientation on $X$.

Again, the fact that $\operatorname{Map}(X, F)$ is a derived Artin stack when $F$ is one, can be deduced from Lurie's version of Artin representability criterion. We will be mainly interested in the special case where $F$ is either a smooth quasi-projective variety, or a classifying stack $B G$, or the derived stack of perfect complexes $\mathbb{R}$ Perf. In all these specific situations, the fact that $\operatorname{Map}(X, F)$ is a derived Artin stack locally of finite presentation can be found in [HAG-II, $§ 2.2 .6 .3$ ] and [Si2].

The Dolbeault and de Rham complexes can also be considered together at the same time, by taking $X:=Y_{H o d} \rightarrow \mathbb{A}^{1}$, see Si1]. More generally, we could take $X$ to be any nice enough formal groupoid (as in [Si1]).

- Mapping stacks with Calabi-Yau source. Let now $X$ be a smooth and proper Deligne-Mumford stack over Spec $k$ of relative dimension $d$, with connected geometric fibers. We assume that we are given an isomorphism of line bundles

$$
u: \omega_{X / k}=\wedge^{d} \Omega_{X / k}^{1} \simeq \mathcal{O}_{X} \equiv \mathcal{O} .
$$

Considered as a derived stack $X$, is automatically $\mathcal{O}$-compact. Moreover, the isomorphism $u$, together with the trace map, defines an isomorphism

$$
H^{d}(X, \mathcal{O}) \stackrel{u}{\longrightarrow} H^{d}\left(X, \omega_{X / k}\right) \stackrel{t r}{\longrightarrow} k .
$$

This isomorphism induces a well defined morphism of complexes

$$
C(X, \mathcal{O}) \longrightarrow k[-d]
$$

which, by Serre duality, is indeed an $\mathcal{O}$-orientation on $X$.

As above, the fact that $\operatorname{Map}(X, F)$ is a derived Artin stack when $F$ is one, can be deduced from Lurie's version of Artin representability criterion. We will be mainly 
interested in the special case where $F$ is either a smooth quasi-projective variety, or a classifying stack $B G$, or the derived stack of perfect complexes $\mathbb{R}$ Perf. In all these specific situations, the fact that $\operatorname{Map}(X, F)$ is a derived Artin stack locally of finite presentation can be found in [HAG-II, $§ 2.2 .6 .3$ ] and [To-Va].

We gather the following consequences of Theorem 2.5 and of the examples above, in the following

Corollary 2.6 Let $G$ be a reductive affine group scheme over Spec $k$. Let $Y$ be a smooth and proper Deligne-Mumford stack over Spec $k$ with connected geometric fibers of relative dimension d. Assume that we have fixed a non-degenerate $G$-invariant symmetric bilinear form on $\mathfrak{g}$.

1. The choice of a fundamental class $[Y] \in H_{D R}^{2 d}(Y, \mathcal{O})$ determines a canonical $2(1-d)$ shifted symplectic form on the derived stack

$$
\mathbb{R} L o c_{D R}(Y, G):=\operatorname{Map}\left(Y_{D R}, B G\right)
$$

of flat G-bundles on $Y$.

2. The choice of a fundamental class $[Y] \in H_{\text {Dol }}^{2 d}(Y, \mathcal{O})$ determines a canonical $2(1-d)$ shifted symplectic form on the derived stack

$$
\mathbb{R} L o c_{D o l}(Y, G):=\operatorname{Map}\left(Y_{D o l}, B G\right)
$$

of Higgs G-bundles on $Y$.

3. When it exists, the choice of a trivialization isomorphism $\omega_{Y / k} \simeq \mathcal{O}_{Y}$, determines a canonical $(2-d)$-shifted symplectic form on the derived stack of $G$-bundles on $Y$

$$
\mathbb{R} B u n(Y, G):=\operatorname{Map}(Y, B G)
$$

of $G$-bundles on $Y$.

4. If $M$ is a compact, orientable topological manifold of dimension d, then a choice of a fundamental class $[M] \in H_{d}(M, k)$ determines a canonical $(2-d)$-shifted symplectic form on the derived stack

$$
\mathbb{R} \operatorname{Loc}(M, G):=\operatorname{Map}(M, B G)
$$

of local systems of principal $G$-bundles on $M$.

In section 3 we will explain how these $n$-shifted symplectic structures compare with the well known symplectic structures on certain coarse moduli spaces (e.g. on character varieties, moduli spaces of stable sheaves on K3-surfaces, etc.). 


\subsection{Lagrangian intersections}

We will be interested here in the study of derived symplectic structures induced on fiber products of derived Artin stacks. In order to do this, we first need to introduce the notion of isotropic and Lagrangian data on a morphism with symplectic target, extending the usual notions of isotropic and Lagrangian sub-varieties of a smooth symplectic manifold. We will show (Theorem 2.9) that the fiber product of two morphisms with Lagrangian structures towards an $n$-shifted symplectic target is naturally equipped with an $(n-1)$-shifted symplectic structure. In particular, the derived intersection of two smooth (algebraic) usual Lagrangians in a smooth (algebraic) symplectic manifold carries a canonically induced (-1)-shifted symplectic structure.

We fix a derived Artin stack $F$ and an $n$-shifted symplectic form $\omega \in \mathbb{S} y m p(F, n)$ on $F$. For $f: X \longrightarrow F$ a morphism of derived Artin stack, we have the pull-back closed 2-form

$$
f^{*}(\omega) \in \mathcal{A}^{2, c l}(X, n)
$$

Definition 2.7 An isotropic structure on $f$ (relative to $\omega$ ) is a path between 0 and $f^{*}(\omega)$ in the space $\mathcal{A}^{2, c l}(X, n)$. The space of isotropic structures on $f$ (relative to $\omega$ ) is defined to be the path space

$$
\operatorname{Isot}(f, \omega):=\operatorname{Path}_{0, f^{*}(\omega)}\left(\mathcal{A}^{2, c l}(X, n)\right) .
$$

A Lagrangian structure on the morphism $f$ (with respect to $\omega$ ) will be an isotropic structure satisfying some non-degeneracy condition. To introduce this condition, let us fix an isotropic structure $h \in \operatorname{Isot}(f, \omega)$. We consider the 2 -form $\mathbb{T}_{F} \wedge \mathbb{T}_{F} \longrightarrow \mathcal{O}_{F}[n]$ underlying $\omega$, as well as its pull-back on $X, f^{*}\left(\mathbb{T}_{F}\right) \wedge f^{*}\left(\mathbb{T}_{F}\right) \longrightarrow \mathcal{O}_{X}[n]$. By definition, $h$ gives us a homotopy between 0 and the composite morphism

$$
\mathbb{T}_{X} \wedge \mathbb{T}_{X} \longrightarrow f^{*}\left(\mathbb{T}_{F}\right) \wedge f^{*}\left(\mathbb{T}_{F}\right) \longrightarrow \mathcal{O}_{X}[n] .
$$

We let $\mathbb{T}_{f}$ be the relative tangent complex of $f$, so that we have an exact sequence of perfect complexes on $X$

$$
\mathbb{T}_{f} \longrightarrow \mathbb{T}_{X} \longrightarrow f^{*}\left(\mathbb{T}_{F}\right) .
$$

The isotropic structure $h$ induces also a homotopy between 0 and the composite morphism

$$
\mathbb{T}_{f} \otimes \mathbb{T}_{X} \longrightarrow \mathbb{T}_{X} \wedge \mathbb{T}_{X} \longrightarrow f^{*}\left(\mathbb{T}_{F}\right) \wedge f^{*}\left(\mathbb{T}_{F}\right) \longrightarrow \mathcal{O}_{X}[n]
$$

As the morphism $\mathbb{T}_{f} \longrightarrow f^{*}\left(\mathbb{T}_{F}\right)$ comes itself with a canonical homotopy to 0 , by composing these homotopies, we end up with a loop pointed at 0 in the space $\operatorname{Map}_{L_{\text {qcoh }}(X)}\left(\mathbb{T}_{f} \otimes \mathbb{T}_{X}, O_{X}[n]\right)$. This loop defines an element in

$$
\pi_{1}\left(\operatorname{Map}_{L_{\text {qcoh }}(X)}\left(\mathbb{T}_{f} \otimes \mathbb{T}_{X}, \mathcal{O}_{X}[n]\right), 0\right) \simeq\left[\mathbb{T}_{f} \otimes \mathbb{T}_{X}, \mathcal{O}_{X}[n-1]\right] .
$$

By adjunction, we get a morphism of perfect complexes on $X$

$$
\Theta_{h}: \mathbb{T}_{f} \longrightarrow \mathbb{L}_{X}[n-1]
$$

depending on the isotropic structure $h$. 
Definition 2.8 Let $f: X \longrightarrow F$ be a morphism of derived Artin stacks and $\omega$ an $n$ shifted symplectic form on $F$. An isotropic structure $h$ on $f$ is a Lagrangian structure on $f$ (relative to $\omega$ ) if the induced morphism

$$
\Theta_{h}: \mathbb{T}_{f} \longrightarrow \mathbb{L}_{X}[n-1]
$$

is a quasi-isomorphism of perfect complexes.

The usefulness of Lagrangian structures is shown by the following existence theorem.

\section{Theorem 2.9 Let}

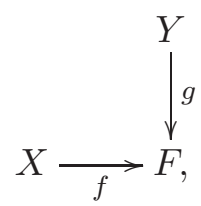

be a diagram of derived Artin stacks, $\omega \in \mathbb{S} y m p(F, n)$ an $n$-shifted symplectic form on $F$, and $h$ (respectively, $k$ ) be a Lagrangian structure on $f$ (respectively, on $g$ ). Then, the derived Artin stack $X \times{ }_{F}^{h} Y$ is equipped with a canonical $(n-1)$-shifted symplectic structure called the residue of $\omega$, and denoted by $R(\omega, h, k)$.

Proof. Let $Z:=X \times_{F}^{h} Y$. The two morphisms

$$
p: Z \longrightarrow X \longrightarrow F \quad q: Z \longrightarrow Y \longrightarrow F
$$

come equipped with a natural homotopy $u: p \Rightarrow q$. This $u$ gives rise to a homotopy between the induced morphisms on the spaces of closed 2-forms

$$
u^{*}: p^{*} \Rightarrow q^{*}: \mathcal{A}^{2, c l}(X, n) \longrightarrow \mathcal{A}^{2, c l}(Z, n) .
$$

Moreover, $h$ (respectively, $k$ ) defines a path in the space $\mathcal{A}^{2, c l}(Z, n)$

$$
h: 0 \leadsto p^{*}(\omega)
$$

(respectively,

$$
\left.k: 0 \leadsto q^{*}(\omega) .\right)
$$

By concatenation of $h, u^{*}(\omega)$ and $k^{-1}$, we get a loop at 0 in the space $\mathcal{A}^{2, c l}(Z, n)$, therefore a well defined element

$$
R(\omega, h, k) \in \pi_{1}\left(\mathcal{A}^{2, c l}(Z, n)\right) \simeq \pi_{0}\left(\mathcal{A}^{2, c l}(Z, n-1)\right) .
$$

It remains to show that this closed 2-form of degree $(n-1)$ is non-degenerate. This follows from the definition of a Lagrangian structure. To see this, let $\pi:=p: Z \longrightarrow F$ be the natural map6, and

$$
p r_{X}: Z \longrightarrow X \quad p r_{Y}: Z \longrightarrow Y
$$

\footnotetext{
${ }^{6}$ Since we just want to prove that the closed 2 -form is non-degenerate, we might equivalently have chosen to run the argument for $\pi:=q$, instead of $\pi:=p$.
} 
the two projections. By definition we have a commutative diagram in $L_{q c o h}(Z)$ with exact rows

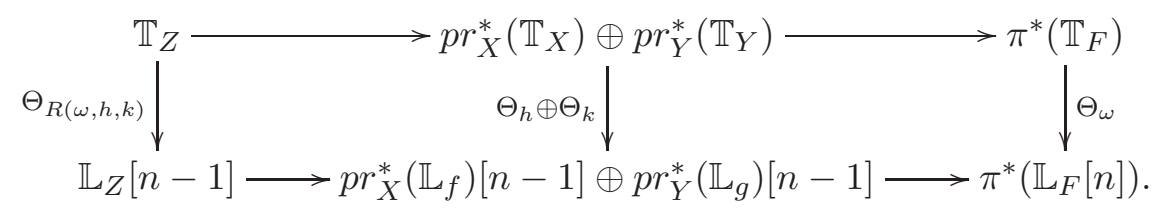

The morphism $\Theta_{\omega}$ is a quasi-isomorphism because $\omega$ is non-degenerate. The morphism $\Theta_{h} \oplus \Theta_{k}$ is also a quasi-isomorphism because of the definition of Lagrangian structures. This implies that $\Theta_{R(\omega, h, k)}$ is a quasi-isomorphism, and thus that $R(\omega, h, k)$ is an $(n-1)$ shifted symplectic structure.

An immediate corollary is the following statement.

Corollary 2.10 Let $X$ be a smooth Deligne-Mumford stack over Speck, $\omega$ a symplectic form on $X$, and $L, L^{\prime} \subset X$ two smooth closed Lagrangian substacks (in the sense that $\omega$ vanishes on $L$ and $L^{\prime}$, and both $L$ and $L^{\prime}$ are of middle dimension). Then, the derived fiber product $L \times_{X}^{h} L^{\prime}$ carries a canonical (-1)-shifted symplectic structure.

Proof. It follows by the following simple observations. As $X, L$ and $L^{\prime}$ are smooth, the spaces of closed 2 -forms of degree 0 on $X, L$ and $L^{\prime}$ are (homotopically) discrete (and equal to $\left.H^{0}\left(X, \Omega_{X / k}^{2, c l}\right), H^{0}\left(L, \Omega_{L / k}^{2, c l}\right), H^{0}\left(L^{\prime}, \Omega_{L^{\prime} / k}^{2, c l}\right)\right)$. From this it follows that the spaces of isotropic and Lagrangian structures on the two inclusions

$$
L \hookrightarrow X \quad L^{\prime} \hookrightarrow X
$$

are either empty or (equivalent to) a point. As $L$ and $L^{\prime}$ are Lagrangian substacks it is easy to see that these spaces are non-empty and hence both equivalent to a point. In particular, there are unique Lagrangian structures on the above two inclusion morphisms, and thus Theorem 2.9 implies that $L \times_{X}^{h} L^{\prime}$ is endowed with a canonical (-1)-shifted symplectic structure.

A particular case of the above corollary is the existence of $(-1)$-shifted symplectic forms on derived critical loci of functions on smooth Deligne-Mumford stacks (see also [Ve] for a more direct local approach).

Corollary 2.11 Let $X$ be a smooth Deligne-Mumford stack over Spec $k$, and $f \in \mathcal{O}(X)$ a global function on $X$, with differential $d f: X \longrightarrow T^{*} X$. Then, the derived critical locus of $f$, defined as the derived fiber product

$$
\mathbb{R} C r i t(f):=X \times_{d f, T^{*} X, 0} X,
$$

of the zero section with the section $d f$ inside the total cotangent stack $T^{*} X$, carries a canonical (-1)-shifted symplectic structure.

Proof. We simply observe that $T^{*} X$ carries a canonical symplectic structure and that $X$ sits inside $T^{*} X$ as two Lagrangian substacks, either via the zero section of via the section $d f$. 


\section{$2.3 \quad 2$-shifted symplectic structure on $\mathbb{R}$ Perf}

We now state our third existence theorem, giving a canonical 2-shifted symplectic structure on the derived stack of perfect complexes $\mathbb{R}$ Perf. This 2-shifted symplectic form will be constructed using the Chern character of the universal object on $\mathbb{R}$ Perf, with values in negative cyclic homology. We will use the construction in [To-Ve-1], as it is perfectly suited to our context, but any functorial enough construction of the Chern character could be used instead.

We recall from [To-Va] the definition of the derived stack $\mathbb{R}$ Perf, which was denoted there by $\mathcal{M}_{\mathbf{1}}$. The functor $\mathbb{R}$ Perf sends a differential non-positively graded algebra $A$ to the nerve of the category of perfect (i.e. homotopically finitely presentable, or equivalently, dualizable in the monoidal model category of $A$-dg-modules) $A$-dg-modules which are cofibrant in the projective model structure of all $A$-dg-modules. It is a locally geometric derived stack, that is a union of open substacks which are derived Artin stacks of finite presentation over Spec $k$. Everything we said about derived Artin stacks locally of finite presentation also make sense for $\mathbb{R}$ Perf, in particular we can speak about $p$-forms, closed $p$-forms and symplectic structures on $\mathbb{R}$ Perf, even though $\mathbb{R}$ Perf is not strictly speaking a derived Artin stack.

On the derived stack $\mathbb{R}$ Perf we have the universal perfect complex $\mathcal{E} \in L_{\text {parf }}(\mathbb{R}$ Perf $)$. The endomorphisms of this perfect complexes define a perfect dg-algebra over $\mathbb{R}$ Perf, denotes by

$$
\mathcal{A}:=\mathbb{R} \underline{\mathcal{H o m}}(\mathcal{E}, \mathcal{E}) \simeq \mathcal{E}^{\vee} \otimes \mathcal{E} .
$$

The derived loop stack of $\mathbb{R}$ Perf

$$
\mathcal{L} \mathbb{R} \text { Perf }:=\operatorname{Map}\left(S^{1}, \mathbb{R} \text { Perf }\right) \longrightarrow \mathbb{R} \text { Perf }
$$

can be identified with the derived group stack over $\mathbb{R P e r f}$ of invertible elements in $\mathcal{A}$

$$
\mathcal{L} \mathbb{R} \operatorname{Perf} \simeq \mathcal{A}^{*}:=G L_{1}(\mathcal{A}),
$$

where $G L_{1}(\mathcal{A})$ is the group stack of auto-equivalences of $\mathcal{A}$ considered as an $\mathcal{A}$-module. As $\mathcal{A}$ is a perfect dg-algebra over $\mathbb{R}$ Perf, we see that $\mathcal{A}^{*}$ is a derived Artin group stack over $\mathbb{R}$ Perf, and that the corresponding quasi-coherent dg-Lie algebra over $\mathbb{R}$ Perf is $\mathcal{A}$ itself, endowed with its natural bracket structure given by the commutator. This implies that we have a natural quasi-isomorphism of perfect complexes over $\mathbb{R}$ Perf

$$
\mathbb{T}_{\mathbb{R} \text { Perf }} \simeq \mathcal{A}[1] .
$$

This identification can be used to define a 2 -form of degree 2 on $\mathbb{R}$ Perf

$$
\mathbb{T}_{\mathbb{R} \text { Perf }} \wedge \mathbb{T}_{\mathbb{R} \text { Perf }} \simeq \operatorname{Sym}^{2}(\mathcal{A})[2] \longrightarrow \mathcal{O}_{\mathbb{R} \text { Perf }}[2],
$$

which, by definition, is (the shift by 2 of) the composition

$$
\mathcal{A} \otimes \mathcal{A} \stackrel{\text { mult }}{\longrightarrow} \mathcal{A} \stackrel{T r}{\longrightarrow} \mathcal{O}_{\mathbb{R} \text { Perf }},
$$

of the multiplication and the trace (or evaluation) morphism

$$
\mathcal{A} \simeq E^{\vee} \otimes E \longrightarrow \mathcal{O}_{\mathbb{R} \text { Perf }}
$$


This 2 -form is clearly non-degenerate. We will now see that this 2 -form of degree 2 on $\mathbb{R}$ Perf is the underlying 2 -form of a canonical 2-shifted symplectic structure on $\mathbb{R}$ Perf.

We consider two derived stacks

$$
|N C|: \mathbf{d A f f}_{k}^{o p} \longrightarrow \mathbb{S} \quad H C^{-}: \mathbf{d} \mathbf{A f f}_{k}^{o p} \longrightarrow \mathbb{S}
$$

defined as follows. We have the derived stack in mixed complexes DR (we forget the extra grading here), on which we can apply the construction $N C$ to get a derived stack in complexes of $k$-modules. The derived stack $|N C|$ is obtained by applying the $\infty$-functor $E \mapsto|E|$ (i.e. the Dold-Kan construction) to turn $N C$ into a derived stack of spaces. In other words, $|N C|$ sends a commutative $k$-dg-algebra $A$ to the simplicial set obtained from the complex $N C(\mathbf{D R}(A))$.

The derived stack $H C^{-}$sends an affine derived scheme $X$ to $\mathcal{O}(\mathcal{L} X)^{h S^{1}}$, the space of $S^{1}$-homotopy fixed functions on the loop space of $X$. It is easy to describe this derived stack algebraically using simplicial commutative algebras. To a commutative $k$-dg-algebra $A$ we form $A^{\prime}$ the corresponding commutative simplicial $k$-algebra (see [To-Ve-2]), and consider $S^{1} \otimes_{k}^{\mathbb{L}} A^{\prime}$, which is another simplicial commutative algebra on which the simplicial group $S^{1}=B \mathbb{Z}$ acts. The space of homotopy fixed point of this action is a model for $H C^{-}(\operatorname{Spec} A)$

$$
H C^{-}(\operatorname{Spec} A) \simeq\left(S^{1} \otimes_{k}^{\mathbb{L}} A^{\prime}\right)^{h S^{1}} .
$$

The main theorem of [To-Ve-2] states that these two derived stacks $|N C|$ and $H^{-}$ are naturally equivalent (and that there is moreover a unique equivalence respecting the multiplicative structures). Furthermore, the Chern character construction of [To-Ve-1, $\S 4.2]$ produces a morphism of derived stacks $C h: \mathbb{R}$ Perf $\longrightarrow H C^{-}$, and thus, by the mentioned equivalence, a morphism

$$
C h: \mathbb{R P e r f} \longrightarrow|N C| \text {. }
$$

This is the Chern character of the universal perfect complex, and defines a natural element in

$$
C h(\mathcal{E}) \in \pi_{0}(\operatorname{Map}(\mathbb{R} \text { Perf },|N C|)) \simeq H^{0}(N C(\mathbb{R} \text { Perf })) .
$$

We can project this element by the projection on the weight 2 piece $N C \longrightarrow N C^{w}(2)$ to get

$$
C h(\mathcal{E})_{2} \in H^{0}\left(N C^{w}(\mathbb{R} \text { Perf })(2)\right) \simeq \pi_{0}\left(\mathcal{A}^{2, c l}(\mathbb{R} \text { Perf }, 2)\right),
$$

which is a closed 2-form of degree 2 .

Theorem 2.12 The closed 2-form $C h(\mathcal{E})_{2}$ defined above is a 2-shifted symplectic structure on $\mathbb{R}$ Perf.

Proof. The proof consists in identifying the underlying 2-form (of degree 2) of $C h(\mathcal{E})_{2}$, and show that it coincides (up to a factor $\frac{1}{2}$ ) with the 2 -form described earlier in this section

$$
\mathcal{A} \otimes \mathcal{A} \stackrel{\text { mult }}{\longrightarrow} \mathcal{A} \stackrel{T r}{\longrightarrow} \mathcal{O}_{\mathbb{R} P \text { erf }}
$$


This identification can be seen as follows. For a perfect complex $E$ on a derived Artin stack $X$, its Chern character $C h(E)$ has an image in Hodge cohomology

$$
C h(E)=\sum C h_{p}(E) \in \oplus_{p} H^{p}\left(X, \wedge^{p} \mathbb{L}_{X / k}\right),
$$

induced by the natural morphism from negative cyclic homology to Hochschild homology (i.e. the projection $N C \rightarrow|\mathbf{D R}|$ ). These Hodge cohomology classes can be described using the Atiyah class of $E$

$$
a_{E}: E \longrightarrow E \otimes_{\mathcal{O}_{X}} \mathbb{L}_{X / k}[1]
$$

We can compose this class with itself to get

$$
a_{E}^{i}: E \longrightarrow E \otimes_{\mathcal{O}_{X}}\left(\wedge^{i} \mathbb{L}_{X / k}\right)[i]
$$

which we write as

$$
a_{E}^{i}: \mathcal{O}_{X} \longrightarrow E^{\vee} \otimes_{\mathcal{O}_{X}} E \otimes_{\mathcal{O}_{X}}\left(\wedge^{i} \mathbb{L}_{X / k}\right)[i]
$$

Composing with the trace $E^{\vee} \otimes_{\mathcal{O}_{X}} E \longrightarrow \mathcal{O}_{X}$, we obtain classes in $H^{i}\left(X, \wedge^{i} \mathbb{L}_{X / k}\right)$. We have

$$
C h_{i}(E)=\frac{\operatorname{Tr}\left(a_{E}^{i}\right)}{i !} \in H^{i}\left(X, \wedge^{i} \mathbb{L}_{X / k}\right)
$$

We come back to our specific situation where $X=\mathbb{R}$ Perf and $E=\mathcal{E}$ is the universal perfect complex. The shifted cotangent complex $\mathbb{L}_{X / k}[1]$ is naturally equivalent to $\mathcal{E} \otimes_{\mathcal{O}_{X}}$ $\mathcal{E}^{\vee}$, and the Atiyah class

$$
a_{\mathcal{E}}: \mathcal{E} \longrightarrow \mathcal{E} \otimes \mathcal{O}_{X} \mathcal{E} \otimes \mathcal{O}_{X} \mathcal{E}^{\vee}
$$

is simply the adjoint of the multiplication morphism

$$
\mathcal{E} \otimes \mathcal{A} \longrightarrow \mathcal{E}
$$

where $\mathcal{A}=\mathbb{R} \underline{\mathcal{H} o m}(\mathcal{E}, \mathcal{E})$ is the endomorphism dg-algebra of $\mathcal{E}$. From this we get the required formula

$$
C h_{2}(\mathcal{E})=\frac{1}{2} \cdot \operatorname{Tr}(\text { mult }) \in H^{2}\left(\mathbb{R} \text { Perf }, \wedge^{2} \mathbb{L}_{\mathbb{R} \text { Perf }}\right)
$$

As a corollary of the last theorem and of theorem 2.5, we have the following statement, which is an extension of the corollary 2.6 from the case of vector bundles to the case of perfect complexes.

Corollary 2.13 Let $Y$ be a smooth and proper Deligne-Mumford stack with connected geometric fibers of relative dimension $d$.

1. The choice of a fundament class $[Y] \in H_{D R}^{2 d}(Y, \mathcal{O})$ determines a canonical $2(1-d)$ shifted symplectic form on the derived stack of perfect complexes with flat connexions on $Y$

$$
\mathbb{R} \operatorname{Perf}_{D R}(Y):=\operatorname{Map}\left(Y_{D R}, \mathbb{R} \text { Perf }\right) .
$$


2. The choice of a fundament class $[Y] \in H_{\text {Dol }}^{2 d}(Y, \mathcal{O})$ determines a canonical $2(1-d)$ shifted symplectic form on the derived stack of perfect complexes with Higgs fields

$$
\mathbb{R} \operatorname{Perf}_{D o l}(Y):=\operatorname{Map}\left(Y_{D o l}, \mathbb{R} \text { Perf }\right) .
$$

3. The choice of a trivialization (when it exists) $\omega_{Y / k} \simeq \mathcal{O}_{Y}$, determines a canonical $2-d$-shifted symplectic form on the derived stack of perfect complexes on $Y$

$$
\mathbb{R} \operatorname{Perf}(Y):=\operatorname{Map}(Y, \mathbb{R P e r f}) .
$$

4. If $M$ is a compact, orientable topological manifold of dimension d, then a choice of a fundamental class $[M] \in H_{d}(M, k)$ determines a canonical $(2-d)$-shifted symplectic form on the derived stack of perfect complexes on $M$

$$
\mathbb{R} \operatorname{Perf}(M):=\operatorname{Map}(M, \mathbb{R P e r f})
$$

\section{$3 \quad$ Examples and applications}

We present in this last section some examples and consequences of our results.

\subsection{0-shifted symplectic structures on moduli of sheaves on curves and surfaces}

We start here by explaining how theorems 2.5 and 2.12 can be used in order to recover the existence of well known symplectic forms on certain moduli spaces of local systems on curves, and vector bundles (or more generally of perfect complexes) on K3 and abelian surfaces.

Local systems on curves. To start with, assume that $G$ is a simple algebraic group over some field $k$, and $C$ be a (geometrically connected) smooth and proper curve over $k$. We have the derived moduli stacks $\mathbb{R L o c}{ }_{D R}(C, G), \mathbb{R L o c}_{D o l}(C, G)$ and $\mathbb{R L o c}_{B}(C, G):=$ $\mathbb{R L o c}\left(C^{t o p}, G\right)$, of local systems of principal $G$-bundles on $C$, Higgs $G$-bundles on $C$, and flat $G$-bundles on the underlying topological space $C^{\text {top }}$ of $C$. According to our corollary 2.6. a choice of an orientation of $C$ determines 0 -shifted symplectic structures on these spaces. These spaces contain smooth Deligne-Mumford substacks consisting of simple objects

$$
\begin{gathered}
\operatorname{Loc}_{D R}(C, G)^{s} \subset \mathbb{R L o c}_{D R}(C, G) \quad \operatorname{Loc}_{D o l}(C, G)^{s} \subset \mathbb{R L o c}_{D o l}(C, G) \\
\operatorname{Loc}_{B}(C, G)^{s} \subset \mathbb{R L o c}_{B}(C, G) .
\end{gathered}
$$

These substacks of simple objects are moreover étale gerbes over smooth algebraic varieties, bounded by the center of $G$ (so they are algebraic varieties as soon as this center is trivial). Therefore, the restriction of these 0-shifted symplectic forms on these substacks define symplectic forms on the corresponding smooth algebraic varieties. We recover this way well known symplectic structures on the coarse moduli space of simple flat $G$-bundles, simple Higgs $G$-bundles, and simple flat $G$-bundles on $C^{\text {top }}$ (see e.g. [Go, Je, In-Iw-Sa]). 
It is interesting to note here that our results imply that these symplectic forms existing on the smooth locus of simple objects have canonical extension to the whole derived moduli stacks. Another interesting remark is the case of $C=\mathbb{P}^{1}$ is a projective line. The corresponding coarse moduli of simple objects is just a point, but the derived stacks are non-trivial. For instance, in the de Rham setting, we have

$$
\mathbb{R L o c}_{D R}(C, G) \simeq\left[\left(\operatorname{Spec} \operatorname{Sym}\left(\mathfrak{g}^{\vee}[1]\right)\right) / G\right] .
$$

This derived stack has already been considered in [La], and according to our results carries a canonical 0 -shifted symplectic form. The tangent complex at the unique closed point is

$$
\mathbb{T} \simeq \mathfrak{g}[1] \oplus \mathfrak{g}[-1]
$$

and the 0 -shifted symplectic form there is the canonical identification

$$
\mathfrak{g}[1] \oplus \mathfrak{g}[-1] \simeq \mathfrak{g}^{\vee}[1] \oplus \mathfrak{g}^{\vee}[-1]
$$

induced by the isomorphism $\mathfrak{g} \simeq \mathfrak{g}^{\vee}$ given by the data of the (essentially unique) symmetric, non-degenerate, bilinear $G$-invariant form on $\mathfrak{g}$.

Perfect complexes on CY surfaces. As a second example we let $S$ be a Calabi-Yau surface (either a K3 or an abelian surface) over a field $k$, equipped with a trivialization $\omega_{S} \simeq \mathcal{O}_{S}$. We have $\mathbb{R} \operatorname{Perf}(S)$, the derived moduli stack of perfect complexes on $S$. According to our corollary 2.13, this derived stack is equipped with a canonical 0-shifted symplectic form. We let $\mathbb{R} \operatorname{Perf}(S)^{s} \subset \mathbb{R} \operatorname{Perf}(S)$ be the derived open substack of simple objects, that is perfect complexes with no negative self extensions and only scalar multiplication as endomorphisms (see [To-Va]). We denote by $\mathcal{M}_{S}^{s}$ its truncation

$$
\mathcal{M}_{S}^{s}:=h^{0}\left(\mathbb{R} \operatorname{Perf}(S)^{s}\right) .
$$

The (underived) stack $\mathcal{M}_{S}^{s}$ is a $\mathbb{G}_{m}$-gerbe over an algebraic space $M_{S}^{s}$, locally of finite presentation over $k$. It is proven in [In that $M_{S}^{s}$ is smooth and comes equipped with a natural symplectic structure. The existence of a non-degenerate 2-form is easy, but there are relatively heavy computations in order to prove that this 2 -form is closed. We will explain how this symplectic structure can be deduced from the 0-shifted symplectic structure on the whole derived stack $\mathbb{R} \operatorname{Perf}(S)$. First of all, the 0 -shifted symplectic form restricts to a 0 -shifted symplectic form on the open $\mathbb{R} \operatorname{Perf}(S)^{s}$. We consider the determinant morphism of [Sch-To-Ve, 3.1]

$$
\text { det }: \mathbb{R} \operatorname{Perf}(S)^{s} \longrightarrow \mathbb{R} \operatorname{Pic}(S),
$$

where $\mathbb{R} \operatorname{Pic}(S)$ is the derived Picard stack of $S$, defined to be $\operatorname{Map}_{\mathbf{d S t}_{k}}\left(S, B \mathbb{G}_{m}\right)$. As explained in [Sch-To-Ve, 4.2] there is a natural projection $\mathbb{R} P i c(S) \longrightarrow$ Spec $k\left[e_{1}\right]$, with $e_{1}$ of degree -1 . The choice of a point $s \in S(k)$ (assume there is one for simplicity) defines by pull-back along $s: \operatorname{Spec} k \longrightarrow S$, another projection $\mathbb{R} \operatorname{Pic}(S) \longrightarrow \mathbb{R} \operatorname{Pic}(\operatorname{Spec} k)=B \mathbb{G}_{m}$. These two projections, pre-composed with the determinant map, defines a morphism of derived stacks

$$
\pi: \mathbb{R} \operatorname{Perf}(S)^{s} \longrightarrow \operatorname{Spec} k\left[e_{1}\right] \times B \mathbb{G}_{m}
$$


It is easy to see that this projection is smooth and representable by an algebraic space. Thus, its fiber at the natural base point is a smooth algebraic space $X$, equipped with a natural morphism

$$
j: X \longrightarrow \mathbb{R} \operatorname{Perf}(S)^{s} .
$$

By definition, $X$ is naturally isomorphic to $M_{S}^{s}$, the coarse moduli of the truncation $\mathcal{M}_{S}^{s}$ of $\mathbb{R} \operatorname{Perf}(S)^{S}$. Finally, the 0-shifted symplectic form on $\mathbb{R} \operatorname{Perf}(S)^{S}$ can be pulled back to $X$ by $j$, and defines a closed 2 -form on $X$. The tangent of $X$ at a point corresponding to a perfect complex $E$ is $E x t^{1}(E, E)$, and this 2 -form is the natural pairing

$$
\operatorname{Ext}^{1}(E, E) \times \operatorname{Ext}^{1}(E, E) \stackrel{\cap}{\longrightarrow} \operatorname{Ext}^{2}(E, E) \stackrel{\operatorname{tr}}{\longrightarrow} \operatorname{Ext}^{2}\left(\mathcal{O}_{S}, \mathcal{O}_{S}\right) \simeq k
$$

and thus is a symplectic form on the smooth algebraic space $X$, which is the one constructed in [I].

Again, the interesting remark here is that this symplectic structure on $M_{S}^{s}$ is induced by a 0 -shifted symplectic structure on the whole derived stack $\mathbb{R} \operatorname{Perf}(S)$, which includes all perfect complexes, and in particular non-simple ones, and even complexes with possibly non trivial negative self-extensions.

Remark 3.1 As the morphism

$$
\pi: \mathbb{R} \operatorname{Perf}(S)^{s} \longrightarrow \operatorname{Spec} k\left[e_{1}\right] \times B \mathbb{G}_{m}
$$

is smooth with fibers $X$, it is locally (for the smooth topology) on $\mathbb{R} \operatorname{Perf}(S)^{s}$ equivalent to the projection

$$
X \times \mathbf{S p e c} k\left[e_{1}\right] \times B \mathbb{G}_{m} \longrightarrow \mathbf{S p e c} k\left[e_{1}\right] \times B \mathbb{G}_{m} .
$$

To be more precise, we have a commutative diagram of derived stacks, with cartesian squares

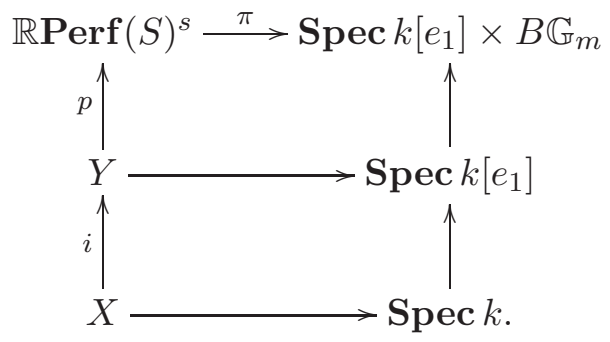

Here, $Y$ and $X$ are derived algebraic spaces, respectively smooth over Spec $k\left[e_{1}\right]$ and Spec $k$. Moreover, $X \simeq h^{0}(Y)$ is the truncation of $Y$, and $i: X \rightarrow Y$ is the natural closed embedding. Locally for the étale topology, $Y$ is a direct product $U \times \operatorname{Spec} k\left[e_{1}\right]$, where $U$ is an étale scheme over $X$. Indeed, any affine scheme $Z$ smooth over $\mathbf{S p e c} k\left[e_{1}\right]$, splits (uniquely, the space of splitting is connected) as a product $Z_{0} \times \operatorname{Spec} k\left[e_{1}\right]$ where $Z_{0}$ is a smooth affine variety. Finally, the morphism $p$ is a $\mathbb{G}_{m}$-torsor and thus is smooth and surjective. This shows that locally for the smooth topology on $\mathbb{R} \operatorname{Perf}(S)^{s}$, the morphism $\pi$ is a direct product. 
Remark 3.2 It is also possible to show that this local decomposition of the previous remark 3.1 is compatible with the symplectic structure. More precisely, locally $\mathbb{R} \operatorname{Perf}(S)^{s}$ looks like $X \times \mathbf{S p e c} k\left[e_{1}\right] \times B \mathbb{G}_{m}$. Now, the derived stack Spec $k\left[e_{1}\right] \times B \mathbb{G}_{m}$ possesses a natural 0 -shifted symplectic structure, for instance by using our corollary 2.6 and the fact that we have a natural identification

$$
\operatorname{Spec} k\left[e_{1}\right] \times B \mathbb{G}_{m} \simeq \mathbb{R M a p}\left(S^{2}, B \mathbb{G}_{m}\right) .
$$

The projection $\pi$ is a symplectic morphism, and the local decomposition

$$
\mathbb{R} \operatorname{Perf}(S)^{s} \simeq X \times \operatorname{Spec} k\left[e_{1}\right] \times B \mathbb{G}_{m}
$$

becomes a decomposition of symplectic derived stacks.

\section{$3.2(-1)$-shifted symplectic structures and symmetric obstruction theo- ries}

We compare here our notion of $(-1)$-shifted symplectic structure with the notion of a symmetric obstruction theory of [Be-Fa, Def. 1.10]. Recall first from [Sch-To-Ve, §1] that for any derived stack $F$ which is locally of finite presentation, its truncation $h^{0}(F)$ comes equipped with a natural perfect obstruction theory. It is constructed by considering the inclusion $j: h^{0}(F) \longrightarrow F$, and by noticing that the induced morphism of cotangent complexes

$$
j^{*}: j^{*}\left(\mathbb{L}_{F / k}\right) \longrightarrow \mathbb{L}_{h^{0}(F)}
$$

satisfies the property to be a perfect obstruction theory. In practice all obstruction theories arise this way.

Assume now that $F$ comes equipped with a $(-1)$-shifted symplectic structure $\omega$. We write the underlying 2-form of degree -1 as a morphism of perfect complexes

$$
\omega: \mathbb{T}_{F} \wedge \mathbb{T}_{F} \longrightarrow \mathcal{O}_{F}[-1]
$$

We use the fact that $\omega$ is non-degenerate and the equivalence

$$
\Theta_{\omega}: \mathbb{T}_{F} \simeq \mathbb{L}_{F}[-1]
$$

to get another morphism

$$
\operatorname{Sym}^{2}\left(\mathbb{L}_{F}\right)[-2] \simeq\left(\mathbb{L}_{F}[-1]\right) \wedge\left(\mathbb{L}_{F}[-1]\right) \simeq \mathbb{T}_{F} \wedge \mathbb{T}_{F} \longrightarrow \mathcal{O}_{F}[-1],
$$

which we rewrite as

$$
\operatorname{Sym}^{2}\left(\mathbb{L}_{F}\right) \longrightarrow \mathcal{O}_{F}[1] \text {. }
$$

This pairing stays non-degenerate, and thus defines a equivalence

$$
\mathbb{L}_{F} \simeq \mathbb{T}_{F}[1]
$$

When restricted to the truncation $h^{0}(F) \hookrightarrow F$, we find that the perfect obstruction theory $E:=j^{*}\left(\mathbb{L}_{F / k}\right)$, comes equipped with a natural equivalence

$$
E \simeq E^{\vee}[1]
$$

which is symmetric (i.e. comes from a morphism $\operatorname{Sym}^{2}(E) \rightarrow \mathcal{O}[1]$ ). It is, by definition [Be-Fa, Def. 1.10], a symmetric obstruction theory on $h^{0}(F)$. 
Remark 3.3 As explained in [Sch-To-Ve], the datum of the truncation $h^{0}(F)$ together with the obstruction theory $j^{*}\left(\mathbb{L}_{F}\right)$ is strictly weaker than the datum of the derived stack $F$. In the same way, the passage from a $(-1)$-shifted symplectic form on $F$ to a symmetric obstruction theory on $h^{0}(F)$ looses important informations. The most important one is that the corresponding symmetric obstruction theory only depends on the underlying 2form of $\omega$, and thus does not see that $\omega$ comes with the important further closedness datum.

An interesting related question is whether a symmetric obstruction theory that is induced by a $(-1)$-shifted symplectic form, is étale locally isomorphic to the canonical one given by lagrangian intersections on a smooth scheme, or even to that existing on the derived zero locus of a closed 1-form on a smooth scheme - instead of just an almost-closed 1form, as in the case of a general symmetric obstruction theory, see [Be, §3.4]. Some very interesting formal and local (for the analytic topology) results in this direction have been proven in $\mathrm{Br}-\mathrm{Bu}-\mathrm{Du}-\mathrm{Jo}$ ] slightly after the appearance of the first preprint version of this paper, and efficiently applied by the same authors to show the existence of local potentials for Donaldson-Thomas theory on Calabi-Yau 3-folds.

Three main sources of examples of (-1)-shifted symplectic structures, and thus of symmetric obstruction theories, are the following.

Sheaves on CY 3-folds. Let $X$ be a smooth and proper CY manifold of dimension 3 , together with a trivialization $\omega_{X / k} \simeq \mathcal{O}_{X}$. It is a $\mathcal{O}$-compact object endowed with an $\mathcal{O}$-orientation of dimension 3, so our theorem 2.5] can be applied. We find in particular that the derived stack $\mathbb{R} \operatorname{Perf}(X)$ of perfect complexes on $X$ is canonically endowed with a $(-1)$-shifted symplectic structure. This defines a symmetric obstruction theory on the truncation $h^{0}(\mathbb{R} \operatorname{Perf}(X))$. The $(-1)$-shifted symplectic structure also induces a $(-1)$-shifted symplectic structure on the derived stack of perfect complexes with fixed determinant. For this, we use the determinant map of [Sch-To-Ve]

$$
\text { det }: \mathbb{R} \operatorname{Perf}(X) \longrightarrow \mathbb{R} \operatorname{Pic}(X),
$$

and consider the fiber at a given global point $L \in P i c(X)$, corresponding to a line bundle on $X$

$$
\mathbb{R} \operatorname{Perf}(X)_{L}:=\operatorname{det}^{-1}(\{L\}) .
$$

The (-1)-shifted symplectic form on $\mathbb{R P e r f}(X)$ can be pulled-back to a closed 2-form on $\mathbb{R} \operatorname{Perf}(X)_{L}$ using the natural morphism $\mathbb{R} \operatorname{Perf}(X)_{L} \longrightarrow \mathbb{R} \operatorname{Perf}(X)$. It is easy to see that this closed 2-form stays non-degenerate, and thus defines a $(-1)$-shifted symplectic structure on $\mathbb{R} \operatorname{Perf}(X)_{L}$. Finally, restricting to simple objects, we get a quasi-smooth derived stack $\mathbb{R} \operatorname{Perf}(X)_{L}^{s}$, endowed with a (-1)-shifted symplectic structure. On the truncation $h^{0}\left(\mathbb{R} \operatorname{Perf}(X)_{L}^{s}\right)$ we thus find a perfect symmetric obstruction theory of amplitude $[-1,0]$.

We could also consider similar examples, like $\mathbb{R L o c}(M, G)$ - for $M$ an oriented compact 3-dimension topological manifold, and $G$ a reductive group scheme over $k$ - or $\mathbb{R} \operatorname{Perf}(M):=\mathbb{R} \operatorname{Map}(M, \mathbb{R} \operatorname{Perf})$.

Maps from elliptic curves to a symplectic target. The second source of examples of $(-1)$-shifted symplectic structures is by considering maps from an elliptic curve towards 
a symplectic smooth target. Let $E$ be a fixed elliptic curve endowed with a trivialization $\omega_{E / k} \simeq \mathcal{O}_{E}$, and $(X, \omega)$ a smooth symplectic variety over $k$ (for instance $X$ can be $T^{*} Z$ for some smooth $Z$, with its canonical symplectic form. This example is of fundamental interests in elliptic cohomology, see $[\mathrm{Co}-\mathrm{Gw}])$. We let $F:=\mathbb{R} \operatorname{Map}(E, X)$, which by our theorem 2.5 is endowed with a canonical $(-1)$-shifted symplectic structure. The derived stack $F$ is a derived algebraic space, and in fact a quasi-projective derived scheme if $X$ is itself quasi-projective. It is moreover quasi-smooth, as its tangent at a given point $f: E \rightarrow X$ is the Zariski cohomology complex $C^{*}\left(E, f^{*}\left(T_{X}\right)\right)$. We thus have a quasismooth derived algebraic space (or even scheme), endowed with a (-1)-shifted symplectic structure. It gives rise to a symmetric perfect obstruction theory of amplitude $[-1,0]$ on the truncation $h^{0}(F)$. Once again, the existence of the $(-1)$-shifted symplectic form is a stronger statement than the existence of a symmetric obstruction theory. This $(-1)-$ shifted symplectic structure induces, by passing to the derived formal completion at a given point, the degree -1 symplectic structure recently considered by Costello in [Co]. As explained in [Co] it can be used to construct a quantization of the moduli $F$, and for this the datum of the symmetric obstruction theory is not enough.

Lagrangian intersections. Let $(X, \omega)$ be a smooth symplectic scheme over $k$, with two smooth Lagrangian subschemes $L$ and $L^{\prime}$. Then, the two closed immersions $L, L^{\prime} \subset X$ are endowed with a unique Lagrangian structure in the sense of our definition 2.8, and thus our theorem 2.9 implies that the derived intersection $L \times{ }_{X}^{h} L^{\prime}$ carries a natural $(-1)$ shifted symplectic structure. Again this defines a symmetric perfect obstruction theory of amplitude $[-1,0]$ on the truncation, that is on the usual schematic fiber product $L \times_{X} L^{\prime}$. The data of the (-1)-shifted symplectic structure is again stronger than the data of the corresponding symmetric obstruction theory, as for instance it can be used to quantize the intersection $L \times_{X} L^{\prime}$. We will come back to the quantization construction in a future work.

\section{References}

[AKSZ] M. Alexandrov, M. Kontsevich, A. Schwarz, O. Zaboronsky, The geometry of the master equation and topological quantum field theory, Int. J. Modern Phys. A 12 , $14051429,1997$.

[Be] K. Behrend, Donaldson-Thomas type invariants via microlocal geometry, Annals of Mathematics, vol. 170, no. 3 (2009) pp. 1307-1338.

[Be-Fa] K. Behrend, B. Fantechi, Symmetric obstruction theories and Hilbert schemes of points on threefolds, Algebra \& Number Theory, 2 (2008), 313-345.

[Ben-Nad] D. Ben-Zvi, D. Nadler, Loop Spaces and Connections, prépublication arXiv:1002.3636 (2010).

[Ber] J. Bergner, A survey of $(\infty, 1)$-categories, Towards higher categories, 6983, IMA Vol. Math. Appl., 152, Springer, New York, 2010,

[Br-Bu-Du-Jo] C. Brav, V. Bussi, D. Dupont, D. Joyce, Shifted symplectic structures on derived schemes and critical loci, Preprint May 2012. 
[Co] K. Costello, Notes on supersymmetric and holomorphic field theories in dimensions 2 and 4, Preprint, October 2011, available at http://math.northwestern.edu/ costello/sullivan.pdf

[Co-Gw] K. Costello, O. Gwilliam, Factorization algebras in perturbative quantum field theory, Preprint draft, 2011.

[De-Ga] M. Demazure, P. Gabriel, Introduction to algebraic geometry and algebraic groups, North Holland Pub. Co. 1980.

[Fu] B. Fu, A survey on symplectic singularities and symplectic resolutions, Ann. Math. Blaise Pascal 13 (2006), no. 2, 209236.

[Ge] E. Getzler, Batalin-Vilkovisky algebras and two-dimensional topological field theories, Comm. Math. Phys. 159 (1994), 265-285.

[Go] W. Goldman, The symplectic nature of fundamental groups of surfaces, Adv. in Math. 54 (1984), no. 2, 200-225.

[Hu-Le] D. Huybrechts, M. Lehn, The geometry of moduli spaces of sheaves, Aspects of Mathematics, 31, Braunschweig, Vieweg, 1997.

[Il] L. Illusie, Complexe cotangent et déformations I, Lectur Notes in Mathematics 239, Springer Verlag, Berlin, 1971.

[In] M.-A. Inaba, Smoothness of the moduli space of complexes of coherent sheaves on an Abelian or a projective K3 surface, Adv. Math. 227 (2011), no. 4, 1399-1412.

[In-Iw-Sa] M.-A. Inaba, K. Iwasaki, M.-H. Saito, Moduli of Stable Parabolic Connections, Riemann- Hilbert correspondence and Geometry of Painlevé equation of type VI, Publ. Res. Inst. Math. Sci. 42 (2006), no. 4, 987-1089.

[Je] L. Jeffrey, Symplectic forms on moduli spaces of flat connections on 2-manifolds, Geometric topology (Athens, GA, 1993), 268281, AMS/IP Stud. Adv. Math., 2.1, Amer. Math. Soc., Providence, RI, 1997.

[Kal] D. Kaledin, On crepant resolutions of symplectic quotient singularities, Selecta Math. (N.S.) 9 (2003), no. 4, 529-555.

[Kal-Le-So] D. Kaledin, M. Lehn, Ch. Sorger, Singular symplectic moduli spaces, Invent. Math. 164 (2006), no. 3, 591-614.

[Ka] C. Kassel, Cyclic homology, comodules and mixed complexes, Journal of Algebra 107, (1987), 195-216.

[Ke-Lo] B. Keller, W. Lowen, On Hochschild cohomology and Morita deformations, Int. Math. Res. Not. IMRN 2009, no. 17, 32213235.

[Ko-So] M. Kontsevich, Y. Soibelman, Stability structures, motivic Donaldson-Thomas invariants and cluster transformations, Preprint arXiv:0811.2435, 2008. 
[Ku-Ma] A. Kuznetsov, D. Markushevich, Symplectic structures on moduli spaces of sheaves via the Atiyah class, J. Geom. Phys. 59 (2009), no. 7, 843-860.

[La] V. Lafforgue, Quelques calculs reliés à la correspondance de Langlands géométrique sur $\mathbb{P}^{1}$, available at http://www.math.jussieu.fr/ vlafforg/

[Lu1] J. Lurie, Higher Topos Theory, Annals of Mathematics Studies, 170, Princeton University Press, Princeton, NJ, 2009. xviii+925 pp.

[Lu2] J. Lurie, Formal Moduli Problems, Vol. 2, Proceedings of the International Congress of Mathematicians 2010 (R. Bhatia, A. Pal, G. Rangarajan, V. Srinivas, M. Vanninathan editors), World Scientific.

[Lu3] J. Lurie, DAG V,IX, available at http://www.math.harvard.edu/ lurie/

[Lu4] J. Lurie, Quasi-Coherent Sheaves and Tannaka Duality Theorems, available at http://www.math.harvard.edu/ lurie//

[Lu5] J. Lurie, Higher Algebra, available at http://www.math.harvard.edu/ lurie/

[Ma] M. Manetti, Lectures on deformations of complex manifolds, Rendiconti di Matematica 24, (2004), 1-183 (and arxiv:math/0507286).

[Mi-Ri] I. Mirkovic, S. Riche, Linear Koszul duality, Comp. Math. 146 (2010), 233-258.

[Mu] S. Mukai, Symplectic structure of the moduli space of sheaves on an abelian or K3 surface, Invent. Math. 77 (1984) 101-116.

[Na] Y. Namikawa, Equivalence of symplectic singularities, preprint (2011), arXiv:1102.0865.

[Ne-McG] T. Nevins, K. McGerty, Derived equivalence for quantum symplectic resolutions, preprint (2011), arXiv:1108.6267.

[Pa-Th] R. Pandharipande, R. Thomas, Almost closed 1-forms, Preprint arXiv:1204.3958, April 2012.

[Pe] J. Pecharich, The derived Marsden-Weinstein quotient is symplectic, in preparation.

[Sch-To-Ve] T. Schürg, B. Toën, G. Vezzosi, Derived algebraic geometry, determinants of perfect complexes, and applications to obstruction theories for maps and complexes, Journal für die reine und angewandte Mathematik, to appear.

[Si1] C. Simpson, Algebraic aspects of higher nonabelian Hodge theory, Motives, polylogarithms and Hodge theory, Part II (Irvine, CA, 1998), 417-604, Int. Press Lect. Ser., 3, II, Int. Press, Somerville, MA, 2002.

[Si2] C. Simpson, Geometricity of the Hodge filtration on the $\infty$ 8-stack of perfect complexes over $X_{\mathrm{DR}}$, Mosc. Math. J. 9 (2009), no. 3, 665-721.

[Si3] C. Simpson, Homotopy theory of higher categories, to appear in Cambrigde University Press. 
[Ta-Ts] D. Tamarkin, B. Tsygan, Noncommutative differential calculus, homotopy BV algebras and formality conjectures, Methods Funct. Anal. Topology 6 (2000), no. 2, 85-100.

[To1] B. Toën, Derived Azumaya Algebras and generators for twisted derived categories, Invent. Math. 189 (2012), no. 3, 581652.

[To2] B. Toën, Higher and derived stacks: a global overview, Algebraic geometrySeattle 2005. Part 1, 435487, Proc. Sympos. Pure Math., 80, Part 1, Amer. Math. Soc., Providence, RI, 2009.

[To3] B. Toën, Champs affines, Selecta Math. (N.S.) 12 (2006), no. 1, 39135.

[To-Va] B. Toën, M. Vaquié, Moduli of objects in dg-categories, Ann. Sci. de l'ENS Volume 40, Issue 3, Pages 387-444.

[HAG-II] B. Toën, G. Vezzosi, Homotopical algebraic geometry II: Geometric stacks and applications, Mem. Amer. Math. Soc. 193 (2008), no. 902, x+224 pp.

[To-Ve-1] B. Toën, G. Vezzosi, Caractères de Chern, traces équivariantes et géométrie algébrique dérivée, Preprint arXiv:0903.3292, version of February 2011(submitted).

[To-Ve-2] B. Toën, G. Vezzosi, Algèbres simpliciales $S^{1}$-équivariantes, théorie de de Rham et théorèmes HKR multiplicatifs, Compositio Math., in press.

[To-Va-Ve] B. Toën, M. Vaquié, G. Vezzosi, Deformation theory of dg-categories revisited, in preparation.

[Ve] G. Vezzosi, Derived critical loci I - Basics, preprint arXiv:1109.5213.

[Viz] C. Vizman, Induced differential forms on manifolds of functions, Archivum Mathematicum, vol. 47 (2011), pp. 201-215. 